\title{
Using enhanced Number and Brightness to measure protein oligomerization dynamics in live cells
}

Francesco Cutrale ${ }^{1 *}$, Daniel Rodriguez ${ }^{2 *}$, Verónica Hortigüela ${ }^{3 *}$, Chi-Li Chiu* ${ }^{4 *}$, Jason

Otterstrom $^{5}$, Stephen Mieruszynski ${ }^{6}$, Anna Seriola ${ }^{7}$, Enara Larrañaga ${ }^{3}$, Angel Raya ${ }^{7,8,9}$, Melike Lakadamyali ${ }^{5,10}$, Fraser $\mathrm{SE}^{1}+$, Elena Martinez ${ }^{3,8,11 *}$, Ojosnegros $\mathrm{S}^{1,7,3 * \dagger}$

1 University of Southern California, Translational Imaging Center, Molecular and Computational Biology, 1002 West Childs Way Los Angeles, CA 90089

2 Laboratory of Theoretical \& Applied Mechanics (LMTA), Dept of Mechanical Engineering, Universidade Federal Fluminense, Rua Passo da Pátria 156, Niterói, RJ 24210-240, Brazil

3 Institute for Bioengineering of Catalonia (IBEC), The Barcelona Institute of Science and Technology (BIST), c/ Baldiri Reixac 15-21, Barcelona 08028 Spain.

4 Center for Applied Molecular Medicine, University of Southern California, CA, USA

5 ICFO-The Institute of Photonic Sciences, The Barcelona Institute of Science and Technology, 08860 Castelldefels (Barcelona), Spain

6 The Walter and Eliza Hall Institute of Medical Research, 1G Royal Parade, Parkville, VIC 3052, Australia. Department of Medical Biology, University of Melbourne, Parkville, VIC 3052, Australia.

7 Center of Regenerative Medicine in Barcelona (CMRB), Hospital Duran i Reynals, Av. Gran Via 199203, L'Hospitalet de Llobregat, 08908 Barcelona, Spain.

8 Centro de Investigación Biomédica en Red (CIBER), Av. Monforte de Lemos 3-5, Pabellón 11, Planta 0, 28029 Madrid, Spain.

9 Institució Catalana de Recerca i Estudis Avançats (ICREA), 08010 Barcelona, Spain.

10 Perelman School of Medicine, Department of Physiology, University of Pennsylvania, Clinical Research Building, 415 Curie Boulevard, Philadelphia, PA 19104, USA.

11 Department of Electronics and Biomedical Engineering, University of Barcelona (UB), c/Martí i Franquès 1-11, 08028 Barcelona, Spain

* Equal contribution

+ Corresponding authors:

Scott E. Fraser, Provost Professor of Biology and Bioengineering, Elizabeth Garrett Professor of Convergent Biosciences, Director of Science Initiatives, University of Southern California, Translational Imaging Center, 1050 Childs Way 401 Ray R Irani Hall, Los Angeles, CA 90089, +1 213 740-2414, sfraser@provost.USC.edu

Samuel Ojosnegros, Head of the Bioengineering in Reproductive Health laboratory, Institute for Bioengineering of Catalonia (IBEC), The Barcelona Institute of Science and Technology (BIST), c/ Baldiri Reixac 15-21, Barcelona 08028 Spain, sojosnegros@ibecbarcelona.eu 
KEYWORDS oligomer, multimer, aggregate, aggregation, spectroscopy, ephrin, micro-contact printing, total internal reflection microscopy, TIRF, confocal microscopy, Number and Brightness, N\&B, enhanced Number and Brightness, enhanced N\&B, eN\&B,

4 EDITORIAL SUMMARY This Protocol describes enhanced Number and Brightness (eN\&B), an approach that uses fluorescence fluctuation spectroscopy data to directly measure the oligomerisation state and dynamics of fluorescently-tagged proteins in living cells.

TWEET Detecting protein oligomerisation states and dynamics in live cells using enhanced Number and Brightness (eN\&B).

COVER TEASER Detecting oligomerisation dynamics in live cells

\section{Up to three primary research articles where the protocol has been used and/or developed:}

1. Ojosnegros, S. et al. Eph-ephrin signaling modulated by polymerization and condensation of receptors. Proceedings of the National Academy of Sciences of the United States of America 114, 13188-13193, doi:10.1073/pnas.1713564114 (2017).

2. Hortigüela, V. et al. Nanopatterns of Surface-Bound EphrinB1 Produce Multivalent LigandReceptor Interactions That Tune EphB2 Receptor Clustering. Nano letters 18, 629-637, doi:10.1021/acs.nanolett.7b04904 (2018).

\section{Abstract}

Protein dimerization and oligomerization are essential to most cellular functions, yet it remains a challenge to measure the size of these oligomers in live cells, especially when their size changes over time and space. A commonly used approach to study protein aggregates in cells is Number and Brightness (N\&B), a fluorescence microscopy method that is capable of measuring the apparent average number of molecules and their oligomerisation (brightness) in each pixel from a series of fluorescence microscopy images. We have recently expanded this approach in order to allow resampling of the raw data to resolve the statistical weighting of coexisting species within each pixel. This feature makes eN\&B optimal to capture the temporal aspects of protein oligomerization, when a distribution of oligomers shifts towards a larger central size over time. In this Protocol, we demonstrate the application of eN\&B by quantifying receptor clustering dynamics using EMCCD based total internal reflection microscopy (TIRF) imaging. TIRF provides a superior signal-to-noise ratio, but we also provide guidelines on how to implement eN\&B in confocal microscopes. For every time point, eN\&B requires the acquisition of 200 frames and it will take few seconds up to two minutes to complete a single time-point. We provide an eN\&B (and standard N\&B) Matlab software package amenable to any standard confocal or TIRF microscope (http://bioimaging.usc.edu). The software requires a high RAM computer to run $(64 \mathrm{~Gb})$ and includes a photobleaching detrending algorithm, which allows to extend the live imaging for more than an hour.

\section{Introduction}

The physiological function of proteins often involves the controlled assembly into multimeric complexes $^{1-3}$. Protein multimerization or clustering mediates signal transduction in several classes of receptors including tyrosine kinase receptors ${ }^{4,5}$, bacterial chemotactic receptors ${ }^{6}$, or 
neurotransmitter receptors ${ }^{7}$ among many others. The clustering of membrane proteins regulates the strength of cell adhesion in both integrins and cadherins, as well as the formation of higher order structures such as focal adhesions ${ }^{8,9}$. Viral capsids are typically large multimeric structures assembled by the self-association of many copies of a few different proteins ${ }^{10}$. In addition, large structural cellular components are assembled by homo-polymerization of monomers into fibrils or more complex conformations ${ }^{11,12}$. For instance, endocytosis and vesicle transport occur after the formation of pits coated by clathrin homo-polymers ${ }^{13}$.

In many cellular functions, the stoichiometry of the protein aggregates can tune their activity. For instance, oligomers of different sizes can modulate transcription factor affinity for DNA binding sites or the association with different proteins ${ }^{14-18}$. In addition, the uncontrolled selfassembly of proteins can lead to the formation of non-physiological toxic aggregates, such as fibrins or plaques of Tau or $\alpha$-synuclein in Alzheimer's and Parkinson's diseases respectively ${ }^{19-}$ 27. Thus, understanding both the normal function and the pathologic disorders derived from protein self-assembly requires better tools for analyzing the diversity of molecular species assembled during protein aggregation. A wide variety of experimental questions require assays to interrogate the nanoscale organization of protein assemblies. These assays should be capable not only of measuring the stoichiometry of active protein complexes, but also powerful enough to resolve the dynamics of their aggregation in live cells over time ${ }^{28}$. Several imaging techniques can provide quantitative information of the oligomeric state of a protein complex; however, most of them are limited in one of three experimental goals: (i) obtaining the complete temporal sequence of the oligomerization process; (ii) providing the dynamic range required to measure a broad spectrum of oligomeric sizes; (iii) recovering spatial information. The Number and Brightness (N\&B) method uses fluorescence fluctuation spectroscopy data to directly measure the average oligomeric state of proteins in living cells, thereby satisfying all three experimental goals ${ }^{29}$. Here we describe a detailed protocol for our recently developed approach to perform a statistically enhanced $N \& B$ version $(e N \& B)^{28}$. This analysis advances the standard N\&B by providing not only the average oligomeric value but the distribution of oligomers for every pixel in an image during long acquisition periods.

\section{$\mathrm{N} \& \mathrm{~B}$, basic principles and theory}

A challenging question in fluorescence microscopy is how to measure the average number of molecules in an image and how to measure their oligomerization state or brightness. Let us consider an example with two sequences of time-lapse frames containing either four scattered fluorescent monomers or one tetramer. If the intensity changes are analyzed within a pixel, utilizing a simple average of the fluorescence intensities, this will produce indistinguishable results between the two examples (Figure 1). N\&B instead utilizes first and second moments of the intensity distribution ${ }^{30}$, allowing for the discrimination between different oligomerization states (brightness) of molecules. Larger oligomers will show an increased variance resulting from fluctuations of wider amplitude than monomers, ensuing from diffusing aggregates moving in and out of the focal volume. In general terms, the larger the variance, the fewer molecules contribute to the average. Moreover, the brightness analysis can be done simultaneously in all the pixels of an image, procuring oligomerization maps of entire cells on a 
pixel-by-pixel basis. All things considered, N\&B is the ideal method to study oligomerization in proteins which aggregation is spatially heterogeneous.

The original N\&B theory was developed by Qian and Elson for measurements of molecules in solution ${ }^{31,32}$ and was adapted for live-cell studies by Enrico Gratton's laboratory ${ }^{29}$. $N \& B$ is a moment analysis capable of measuring the apparent average number of molecules and their oligomerization state (brightness) in each pixel from a series of fluorescence microscopy images. The ratio of the square of the average (first moment) intensity $\left(\langle k\rangle^{2}\right)$ to the variance (second moment, $\sigma^{2}$ ) is proportional to the average apparent Number of particles (N). The apparent Brightness, B, which represents the molecular oligomerization level, is calculated as the ratio of variance $\left(\sigma^{2}\right)$ to average intensity $(\langle k\rangle)$.

$$
N=\frac{\langle k\rangle^{2}}{\sigma^{2}}
$$

$$
B=\frac{\sigma^{2}}{\langle k\rangle}
$$

The pixel volume covered by images obtained with optical microscopes working on total internal reflection microscopy (TIRF) mode (assuming an illumination height of 100-200 nm) at maximum resolution is in the range of $0.0011 \mu \mathrm{m}^{3}-0.0022 \mu \mathrm{m}^{3}$, respectively. Depending on the protein size, considering physiological concentrations, this volume can harbor tens to hundreds of proteins assembled into different oligomeric states. In standard N\&B all the molecular diversity is summarized in a single average oligomerization value per pixel ranging from the monomer to roughly 100-mer species. The ability to determine oligomerization heterogeneity is limited mainly by the diffusion rate of the proteins and by the capability of the acquisition device to rapidly sample in time and across a wide dynamic range of fluorescence intensity.

\section{eN\&B: Statistical enhancement}

In standard N\&B, for every time-point, $F$ consecutive frames are acquired for the analysis of the fluorescence fluctuations and the calculation of a single oligomerization value in the sequence. A minimum of $F=25$ is advised to achieve enough statistical robustness, although $F=200$ should be used for deeper analysis ${ }^{29}$. If the oligomer population is relatively homogeneous, the average oligomer size obtained with standard N\&B may be an optimal representation of the general oligomerization state of the protein. However, in some cases, a single average value may not represent the diversity of protein complexes assembled in a single pixel. For this reason, we have developed enhanced $N \& B$ (eN\&B). eN\&B sub-samples the entire dataset $F$ using analysis window of length $w=100$, shifting the window one frame at a time in a circular way until the entire dataset is covered. This statistical resampling results in a distribution of oligomeric values per every pixel. 
The Number and Brightness values are recorded per every shift, ensuring the same statistical weight is given to each frame. Hence for each pixel $(i, j)$ using eN\&B we obtain an array of $F$ values of Brightness $B$. Each Brightness arises from a sliding window defined as follows:

4 $B_{e}=\left\{\begin{array}{c}\left.\frac{\sigma^{2}}{\langle k\rangle}\right|_{n} ^{n+w} \text { if } n<F-w \\ \left.\frac{\sigma^{2}}{\langle k\rangle}\right|_{n} ^{F}+\left.\frac{\sigma^{2}}{\langle k\rangle}\right|_{1} ^{w-(F-n)} \text { if } n>F-w\end{array}\right.$

5 where $e$ goes from 1 to $\left.F \cdot \frac{\sigma^{2}}{\langle k\rangle}\right|_{n} ^{n+w}$ is the $B$ arising from the window of length $w$ starting from 6 position $n$ and ending in $n+w$ while $\left.\frac{\sigma^{2}}{\langle k\rangle}\right|_{n} ^{F}+\left.\frac{\sigma^{2}}{\langle k\rangle}\right|_{1} ^{w-(F-n)}$ joins frames from position $n$ to last

7 frame $\mathrm{F}$ and from position 1 until reaching the number $w$. Similarly, we obtain corresponding F values of apparent Number N.

$$
N_{e}=\left\{\begin{array}{c}
\left.\frac{\langle k\rangle^{2}}{\sigma^{2}}\right|_{n} ^{n+w} \text { if } n<F-w \\
\left.\frac{\langle k\rangle^{2}}{\sigma^{2}}\right|_{n} ^{F}+\left.\frac{\langle k\rangle^{2}}{\sigma^{2}}\right|_{1} ^{w-(F-n)} \text { if } n>F-w
\end{array}\right.
$$

The trajectory of the sliding window follows the time sequence of the dataset; therefore the statistical resampling of eN\&B works as a consecutive N\&B measurement with time delay equal to the frame rate. When this process is repeated for different $T$ points (see next section), we obtain a multidimensional matrix of data containing information from $\mathrm{x}$, y pixel position, distribution of apparent Number and apparent Brightness in each pixel, and time.

Simulations. The power of eN\&B analysis depends on multiple factors, most significantly the dynamic range of oligomer sizes, their change in aggregation and their relative abundance, as well as their absolute concentration within a given measurement point. A simulation including two opposed, complex oligomer populations highlights the benefit of the resolving power of eN\&B over standard N\&B. We simulated two scenarios: one with monomers gradually forming oligomers over time (Figure 2a), and one with different oligomers co-existing in solution (Figure $2 b)$. In the first scenario, eN\&B shows the clear advantage of capturing individual oligomerization state (Figure 2c); in the second scenario, the spread of eN\&B delivers an approximation to the actual distribution of oligomer population (Figure $2 \mathrm{~d}$ ).

Photobleaching compensation and time expansion. On the short term time dimension, camera-based N\&B generally works in the msec to sec range ${ }^{33} 34$, which is limited by hardware capabilities of modern microscope cameras. However, in order to time-resolve the formation of high-order aggregates or processes running with slower, larger dynamics, the acquisition of 
images may require longer exposure than that offered by conventional N\&B. When attempting to time-resolve long oligomerization processes through N\&B analyses, the effect of photobleaching interferes with the measurements. To overcome this, we have implemented boxcar filtering algorithms ${ }^{35}$ to detrend the decay of fluorescence intensity during the multiple light exposures in the sequential acquisition, while at the same time keeping the fluctuations intact ${ }^{23,36-39}$. These algorithms are implemented in our eN\&B software and allow to extend the data acquisition up to 10 to 15 sequential time points or even more, depending on the brightness of the original sample and the frame rate (Supplementary Fig. 1). The original work by Hellriegel et al. shows that even with $50 \%$ bleaching (i.e. the final frame average intensity is $50 \%$ of the original frame intensity), boxcar filter helps to recover the correct brightness estimation ${ }^{35}$. Photobleaching can be modelled by an exponential decay ${ }^{37}$ :

$$
f(t)=e^{-\alpha t}
$$

where the exponential coefficient, $\alpha$, indicates the extent of photobleaching, and the effect of boxcar filtering. Large box sizes do not sufficiently correct for the overestimation of $B$, whereas smaller sizes yield to underestimate the brightness. The optimal boxcar size is dependent on bleaching speed. A smaller boxcar size should be used with faster bleaching (larger $\alpha$ ) to optimally recover brightness. In a recent work ${ }^{40}$, exponential filtering detrending permitted to time-resolve the transition of monomer to dimers of a FKBP1-tagged fluorescent protein and corrected images with up to $25 \%$ of bleaching ${ }^{40,41}$. In both cases, boxcar and exponential filtering, selecting the right window size was crucial to correct the bleaching without discarding the actual fluctuation. A boxcar window of 10 frames was chosen in our software because, as described in the original work $^{35}$, in a biological context, this range will not affect the higher frequency fluorescence fluctuation of fast-diffusing species.

\section{Applications of the method}

The oligomerization of a large number of proteins has been revealed through N\&B analysis. Examples demonstrate the applicability of N\&B to a broad variety of protein families, with localization at all major cellular compartments. In the cytosol, N\&B has been used to resolve the oligomerization dynamics of focal adhesion components such as paxillin and actin $8-10,29,42,43$, and the assembly of viral matrix proteins ${ }^{8-10,29,42,43}$. A number of membrane proteins have been subjected to N\&B analysis, such as Annexins or UPAR ${ }^{36,44}$. N\&B has also been applied to the study of signaling pathways, including p75, LRRK2 ${ }^{45-47}$, ErbB1 and ErbB2 receptor tyrosine kinases ${ }^{48}$, and proteins involved in membrane - lipid - dynamics such as dynamin $2^{49-53}$. In the nucleus, N\&B has revealed the ligand-induced aggregation of transcription factors and has been used to discriminate between different oligomer subpopulations ${ }^{20,54,55}$. In addition, N\&B has been used to study how DNA repair proteins bind to the DNA following the recruitment of double strand break factors ${ }^{56}$. N\&B has also been applied to the study of pathogenic aggregation of peptides causing neurodegenerative diseases, such as huntingtin or alpha synuclein ${ }^{24,38,57}$. Fluorescently tagged molecules other than proteins can also be studied by $N \& B$, examples of which include the aggregation of DNA after lipofection ${ }^{58}$. 
In our work, we used eN\&B to study the oligomerization of the EphB2 receptor during $1 \mathrm{~h}$ timelapse measurements following receptor activation. The Eph receptor is a membrane-tethered protein which forms large aggregates upon interaction with its cognate ligand, ephrin ${ }^{59}$. Despite playing a crucial role in neural development, tissue patterning and regeneration, the dynamics of Eph receptor clustering was poorly understood ${ }^{4,60,61}$. We performed eN\&B analysis of fluorescently-tagged Eph to yield data on the receptor's oligomerisation-state over time. The quality of eN\&B data allowed mathematical modelling of receptor clustering and the proposition of a new mechanism for Eph signalling, termed polymerization-condensation ${ }^{28}$. In our experimental setup, Eph-expressing cells were stimulated with the eprhin ligand presented in four different spatial configurations, namely, ligands in solution, micro-printed ligand dimers, micro-printed ligand clusters, and nanopatterned clusters ${ }^{62}$. eN\&B analysis was able to capture sensible variations between the different modes of ligand presentation and retrieved characteristic oligomerization dynamics for each mode.

\section{Comparison with related methods}

Several different methods have been developed that can be used to study the oligomerisation states/dynamics of proteins in vivo. In this section, we will briefly highlight the key alternative approaches and their advantages and disadvantages compared to eN\&B.

Spectroscopy methods. Spectroscopy methods include N\&B and a broad collection of the focal volume (for a comprehensive review, see Ref. ${ }^{63}$ ). Arguably the most popular spectroscopy application is fluorescence correlation spectroscopy (FCS), which is widely used to efficiently measure the diffusion coefficients of fluorescent molecules and the variation in those coefficients due to the presence of different molecular species (i.e. bound or unbound pairs, oligomers etc.). FCS can also be adapted to measure the oligomerization of proteins, provided that proper calibrations are performed ${ }^{64,65}$. FCS typically works on single-pixels (with few exceptions ${ }^{66}$ ), and it may therefore be challenging to capture the full diversity of oligomeric states using this approach.

Photon counting histogram (PCH). PCH was originally developed by Chen et al. ${ }^{67}$ and is the first method that can be used to extract molecular Number and Brightness information from fluorescence fluctuation data. $\mathrm{PCH}$ is capable of resolving heterogeneous molecular populations ${ }^{68}$ and it has been applied to resolve mixed oligomer populations of membrane receptors ${ }^{69}$. The information attainable by $\mathrm{PCH}$ is robust and complete, however, it is limited to single-point detection. It also requires longer data acquisition as well as data analysis time compared to N\&B.

Fluorescence Resonance Energy Transfer (FRET) imaging. FRET imaging is based on the detection of variations in the fluorescence intensity of a protein due to energy transfer to an acceptor protein located in close (nanometer range) proximity. This is very sensitive approach for detecting the interaction of protein pairs, or in qualitative terms, the formation of oligomers. FRET imaging includes a diverse collection of approaches such as sensitized emission, acceptor photobleaching or anisotropy-based homoFRET ${ }^{12,60}$. These approaches show different capability to quantify the stoichiometry of a narrow oligomeric range ${ }^{70}$. The 
most sensitive FRET versions, which include single-molecule detection ${ }^{71,72}$ and fluorescence lifetime imaging microscopy (FLIM) ${ }^{73}$, can be used to quantify a larger range of oligomeric states, but data acquisition is relatively slow (in the order of minutes) and is better suited to capture the dynamics of slow assembly processes such as amyloid aggregation.

5 Other methods. Super resolution microscopy and single-molecule detection can also be used

6 to estimate the number of proteins contained in a complex by counting fluorophore

7 photobleaching steps ${ }^{74,75}$. Intensity-based methods can quantify local concentration of

8 proteins but cannot extract the oligomer size-distribution.

\section{Limitations of eN\&B}

The camera-based eN\&B technique is dependent on the system capabilities to acquire short exposure images while maintaining high collection efficiency (sensor Quantum Yield) and collection rate with low noise. These characteristics will determine the highest protein diffusion rate that can be imaged using this technique ${ }^{76}$. The protein diffusion rate will also determine the ideal time resolution of the consecutive eN\&B measurements. Fast-diffusing proteins will require short exposure times resulting in a very fast 100-200 frame acquisition. For slowdiffusing proteins the camera exposure time will be longer and therefore capturing 200 frames will take a substantial amount of time. Even if a second time point was to be captured right after the first one, there would be a minimum lapse in the order of minutes, between the start of the two consecutive time-points. In extreme cases, where the protein binding kinetics is fast, the amount of clustering occurring during a single $F=200$ acquisition may be substantial. In most cases, however, the characteristic acquisition time will be faster than in standard FCS or FLIM applications.

The characteristic diffusion rate for proteins inside cells, considering different sizes and cell compartments ranges between $30-0.03 \mu \mathrm{m}^{2} \mathrm{~s}^{-177}$. This range can be captured approximately with an exposure time range of $1 \mathrm{~s}$ to $0.05 \mathrm{~ms}$. Most cameras will be able to deal with the slow side without issue, which typically corresponds to protein diffusion rates within membranes. However, acquiring 200 frames at 1s/frame will expose the cell to considerable amount of light, and photobleaching will have to be assessed carefully. The fastest diffusion rates that can be captured by eN\&B will be limited by the both by shortest exposure time and acquisition speed of the camera, which, at the time this protocol is written, for most brands top around $0.5 \mathrm{~ms}$ and 100 frames s$~^{-1}$ respectively. If using confocal scanning microscopes, the single pixel dwell time will be considerably faster, in the order of $\mu \mathrm{s}$. However, point-scanning systems will trade off in the time to scan through an entire image, which will be considerably longer, as well as the sensitivity of the detectors, which peaks at a quantum efficiency (QE) of $45 \%$ (GaAsP), compared to currently available $95 \%$ QE for high sensitivity cameras (EMCCDs and bsiscCMOS). When working with fast diffusing species (small peptides in the cytoplasm), two things need to be considered. First, if the photon budget is low, a short exposure time will not be sufficient to collect enough photons to reach an optimal signal-to-noise ratio. Second, the relative diffusion rate of GFP should be taken into account when fusing this to small peptides.

41 The diffusion rate of GFP in the eukaryotic cytoplasm is about $27 \mu \mathrm{m}^{2} \mathrm{~s}^{-17877}$ and molecular 42 species diffusing faster than GFP may be slowed down when fused. 
We have not performed a formal analysis of the optimal range of oligomers that can be measured with eN\&B. However, theoretical and experimental measurements with the Eph receptor suggests that eN\&B can discriminate oligomers within the 1-to-40-mer range without saturating the intensity signal. Mathematical estimations show that expanding that range up to 100-mer improves the fitting between imaging data and mathematical models ${ }^{28}$, which suggests that eN\&B might be applied to an even broader range of species. However, these evidences are theoretical, and a formal study about the oligomer range is missing in the N\&B field.

The camera exposure time should be set up to meet the log-linear region of the autocorrelation curve obtained during FCS measurements of the monomer so that all proteins moving in and out of the focal volume are captured by the camera (see Experimental Design protein may decrease with the size of the oligomer ${ }^{79,80}$, which implies that given a certain threshold size, the time the camera collects photons will be an oversampling of the actual aggregate dynamics and an artefactual reduction in the number of oscillations (i.e. the larger aggregates may need longer time than the one set up as the camera exposition time, to come in and out of the focal volume). We use, as a rule of thumb, a cut-off of 40-mers as the upper limit for oligomer detection. However, a mathematical fitting of the empirical data from EphB2 oligomerization suggested that establishing 100-mer as the upper detection limit would result in minimum information loss and better fit of the equations compared to the analysis using a 40-mer upper limit ${ }^{28}$. This broad range of detection may be even wider for membrane proteins which diffusion is not affected by the size of the oligomer ${ }^{80}$.

Other important parameters to consider in calibrating and designing the experiments in this protocol are the linearity of the signal output and the dynamic range of the detector. Given a specific setting configuration for the acquisition device (in this case a camera) the measured intensities need to scale linearly with the input photons. The detector's dynamic range will set the limit in capturing the larger intensity fluctuations. If lower molecular brightness labels are used, the number of frames can be increased to reduce statistical noise and spreading of the standard deviation of the Brightness values ${ }^{29}$

The statistical resampling leading to eN\&B tackles the limitation of $N \& B$ in providing any information additional to a weighted mean aggregate size per pixel. eN\&B cannot discriminate perfectly the relative concentrations per oligomer size, but it adds more statistical representability to the estimations than the standard N\&B (Figure 2). The resampling in eN\&B is analogous to the standard analysis done on time series to produce frequency spectra where an ideal spectrum with discrete and separated tonal frequencies is used to create a synthetic signal ${ }^{81,82}$. The result is a spectrum with side bands and aliasing that does not really reproduce the original discrete tones. In order to faithfully reproduce the original spectrum, very long samples with high sampling rates and a completely ergodic series would be necessary. On the other hand, when applied to time series with a broadband (continuous) spectrum, the resampling recovers reasonably well the spectrum even with sub-optimal sampling parameters. Therefore eN\&B is an optimal algorithm to resolve the oligomerization of proteins over time. During most polymerization processes, a broadband distribution of oligomers sequentially moves to a higher central size and dispersity. eN\&B will not unmix oligomers when perfectly 
overlapping in time and space or extract the distribution that exactly mirrors the real one, partially because sampling rates and series are limited by technology. However the distributions will be centered on the dominant oligomers, and oligomers far from them will be gradually underrepresented at some rate. This was found to be better than a single average of the entire population (as in N\&B), and delivered results more consistent with theoreticalmathematical models ${ }^{28}$.

\section{Microscope setup}

9 N\&B has been implemented successfully on multiple types of fluorescence microscopes, both single point scanning systems and full field camera based systems. In particular, single point scanning systems with analog $20,33,56,83$ and photon counting modes were used, both in confocal (single photon) ${ }^{9,24,38,44-46,50,55}$ and two-photon mode ${ }^{8,36,51}$. EMCCD cameras have been used only in TIRF microscopes ${ }^{10,33,34,42,52}$. Other systems have been used for Fluorescence Correlation Spectroscopy (FCS) analysis, hence, in principle, can be used for N\&B and eN\&B. Such systems are Selective Plane Illumination Microscopes ${ }^{84}$ and Spinning Disk confocal microscopes ${ }^{66,85}$. Enhanced versions of such systems, such as Lattice Light Sheet or $2 p$-Spinning Disk, are likely to enable eN\&B.

Here we describe a detailed protocol to perform eN\&B on an EMCCD camera based TIRF microscope because of the superior signal-to-noise ratio offered by such system. TIRF illumination is restricted to a $100-200 \mathrm{~nm}$ region immediately adjacent to the glass-water interface. While the plasma membrane is the ideal compartment for TIRF microscopy, it can also be employed to study cytosolic proteins; it is possible to reach 1-2 $\mu \mathrm{m}$ deep into the sample when imaging slightly below the critical TIRF angle (oblique incidence geometry). This range allows to image actin, tubulin, or even nuclear proteins, although it is important to keep in mind that there will be a contribution from out of focus fluorophores ${ }^{86,87}$.

Following the calibration strategies described in the original Number and Brightness paper ${ }^{29}$, the eN\&B method and software can also be used to analyze data obtained from confocal $^{9,24,38,44-46,50,55}$ and two-photon microscope set-ups in both photon-counting ${ }^{8,36,51}$ and analog mode ${ }^{20,33,56,83}$ as well as light sheet systems ${ }^{84}$.

\section{Experimental Design}

32 Cell culture preparation. For all the experiments, glass bottom dishes (MatTek Corporation, USA) compatible with confocal and TIRF microscopes are firstly coated with a cell adhesive polypeptide. When using a TIRF microscope we recommend to keep the plate brands and model constant for all the experiments. Different brands and models may have different thickness which affects the TIRF angle and the objective working distance. (Sigma-Aldrich, USA) diluted at $0.05 \%(\mathrm{w} / \mathrm{v})$ in PBS for $90 \mathrm{~min}$ at room temperature and then rinsed 3 times with PBS and Milli-Q water. Controls should be carried out to make sure the cell adhesive coating does not affect the protein under study. We found out that Laminin can activate the Eph receptor efficiently. 
At this point, coated dishes can be air-dried and kept at 4 으 $\mathrm{C}$ for a maximum of 24 hours before the next step. In the example described here, EphrinB1-Fc (R\&D Systems Inc., USA) was selected as ligand for the EphB2 receptor and was presented to the cells either in soluble form (Step 11) or immobilized on the substrate through a printing procedure. Control (mock) stimulation were performed on PLL-coated glasses and on glasses with Fc fragment (Jackson ImmunoResearch, UK) printed onto a PLL coating.

\section{Replicates and Controls}

Several controls can help to place the brightness measurements in the context of protein dynamics. Positive controls should use antibodies or molecules to induce the oligomerization of the protein of interest with high efficiency. In a negative control experiment the oligomer should stay unassembled during the entire time-lapse recording. This can be achieved by imaging the cells in the absence of any induction or by using inhibitory drugs. Mutant proteins, such as negative dominants, can be used to calibrate the sensitivity of the method to different oligomerization kinetics. Photobleaching can be quantified by integrating the fluorescence form a single cell at the beginning and the end of either a single timepoint or the entire timelapse. A crrect experimental design should also include sufficient replicas to obtain statistically significant data to compare the different controls or samples.

Instrument calibration. Ideally, image acquisition settings are determined once at the beginning of an experimental project (Steps 1-10) and maintained constant throughout the experimental procedure for consistency ${ }^{33,34}$. The sections below describe steps to determine key camera and illumination settings affecting the fluctuations extracted in eN\&B.

Camera noise calibration. It is important to optimize the camera's dark count and signal-tonoise ratio at multiple pixel read-out rates and with several EM gain settings, respectively (Step 12). This serves to optimize the image acquisition conditions in a trade-off between speed and instrumental background noise. Camera dark current can be measured with the shutter closed and 500 millisecond exposure time (for a description of the choice of exposure see Experimental Design "Protein diffusion and camera exposure calibration"). Recordings of 200 frames should be obtained at several pixel-transfer rates. The dark count histograms obtained are analyzed regarding its mean and standard deviation values, as well as the uniformity across the EMCCD chip. This calibration step aids in identifying excessive differences in pixel noise, hot pixels and unusual noise patterns which might affect the analysis and hence should be excluded.

Gain. We recommend optimizing the EM gain to maximize the signal-to-noise ratio of the images (Step 7). This can be done one time and the result can be used as the standard gain 
value for a particular microscope (even for other applications). This calibration can be done by imaging fluorescent proteins or fluorescently-labeled antibodies adsorbed to a clean glass surface and minimizing the signal's coefficient of variation (see Box 1 for a step-by-step procedure on gain optimization).

Pixel size. In our lab, imaging is performed with a Nikon NSTORM system equipped with a $100 \mathrm{x}$ Apo TIRF NA 1.49 oil-immersion objective and a 1.5x tube lens engaged (Step 6). However other TIRF and confocal microscopes can be used provided hat high NA objectives are used. The pixel size is determined using the following equation:

$P_{s i}=\frac{P_{s c}}{\left(M * r_{l}\right)}$

Where $P_{s i}$ is the pixel size on the image, $P_{S c}$ is the pixel size on the camera sensor, $M$ is the objective magnification and $r_{l}$ is the relay lens. For our setup, we obtain a final pixel size of 106 $\mathrm{nm}$. A small pixel size is essential for measuring signal fluctuations correctly.

Laser. Illuminating laser power should be determined using two empirical criteria (Step 5). The first is to ensure that the fluorescence intensity attributable to the fluorescent construct (in our case Eph-mRuby) is solidly in the middle of the camera's dynamic range (i.e. peak value $\sim 35,000$ digital levels on 16-bit images), while ensuring there are no saturated pixels. The second criterion is to minimize photobleaching during a single 200-frame acquisition such that the final average intensity within an imaged cell shows no more than $\sim 5 \%$ reduction in fluorescence as compared to the average intensity in the first image.

TIRF angle. We routinely use the commercial Nikon set up for stochastic optical reconstruction microscopy (the NSTORM microscope for our experiments. This setup comprises a robust optical design for focusing the illumination laser light onto the back focal plane of the objective to produce TIR. As a result, the position of the TIR focusing lens can be adjusted once and repeatedly used in the same setting to obtain a similar evanescent field over several imaging sessions. In our example, we imaged cells expressing Eph-mRuby that were strongly adhered to the glass surface and well spread, allowing us to select a field of view showing isolated, nonoverlapping cells. The TIR lens position can be adjusted to optimize visualization of: 1) disappearance of intracellular vesicles that transport the labelled membrane protein and 2) increase of detected fluorescence arising from a local field enhancement near the critical angle for the water-glass interface. It is important to ensure that the intensity counts are consistent between experiments with different TIR lens positions. This optimization process is rapid and can easily be performed for each experiment in microscope systems showing lower robustness in TIR lens positioning (Step 10). 
Camera readout mode. The commercial NSTORM microscope we utilize is equipped with an Andor iXon 897 EMCCD camera capable of either $10 \mathrm{MHz}$ readout rate at 14-bit or $1 \mathrm{MHz}$ at 16bit. We use the slower $1 \mathrm{MHz}$ rate to access the larger 16-bit range and obtain the larger

Analog Number and Brightness calibration. Fluorescence microscopes are affected by instrumental noise. As such, the analysis requires a calibration step which is instrument

$B_{\text {imer }}(i)=1+i\left(B_{\text {monomer }}-1\right)$

where $B_{\text {imer }}$ is the brightness of the $\mathrm{i}$-mer, $i$ is the size of oligomer and $B_{\text {monomer }}$ is the a base for the calibration approach described here. A set of dark images contains the information required for eN\&B calibration. Two components can be discerned in the intensity distribution of these dark sets (Figure 3a): one Gaussian part and one exponential part (linear part on log scale). The center of the Gaussian component represents the offset of the system while its standard deviation is the readout noise (Figure $3 b$ ). The exponential component is used to obtain the conversion factor from intensities measured and photons, extracting the slope $S$ of the curve (Figure 3c). The calibration should be performed separately for every experiment, as subtle variations are observed on a day-to-day usage of the instrument. We provide a software packaged with an automated fitting tool for this purpose (http://bioimaging.usc.edu) (Figure 3d) (Step 19).

Monomer brightness calibration. The brightness of single monomers needs to be estimated from samples were the protein exists in its free form in a monomeric state. In our case study with the Eph receptor, we imaged cells that were seeded for $24 \mathrm{~h}$ on PLL coated plates, and had no exposure whatsoever to any cognate ligand. For any other membrane receptors, a similar procedure must be performed, avoiding serum components or coatings that may bind or interfere with the oligomerization of the protein. Excessive overexpression may also trigger self-aggregation of proteins and must be also avoided. For other proteins it is important to identify where the protein is found in a monomeric state ${ }^{29}$. If obtaining a monomeric population of the protein of interest is not possible, a variant with truncated or mutated oligomerization interfaces can be generated, as long as the diffusion rate is similar to the native protein.

Based on the brightness value of the monomer, the brightness of the different oligomers (imer) can be calculated as follows: measured brightness for the monomer. It is important to note that some fluorescent proteins are known to self-aggregate or work as dimers ${ }^{88}$. In order to overcome fluorescent protein- 
induced dimerization artifacts, several monomeric fluorescent proteins have been described ${ }^{89,90} .88$

4 Protein diffusion and camera exposure calibration. It is essential to determine the ideal 5 camera exposure rate so that fluctuations are accurately captured between frames (Steps 8 and 9). This parameter is related to the diffusion rate of the protein of interest, and can be defined using the autocorrelation function (ACF) from FCS analysis (Figure 4). The details on the protein mobility coefficient may be biologically relevant in addition to the oligomerization dynamics resolved by eN\&B. In the interest of space, readers are directed to a number of excellent review and method articles of FCS $^{91-93}$.

An important factor to consider is the fluorescence density of the sample, as FCS works optimally when a low concentration of protein is present. Molecular crowding saturates the focal volume and reduces the amplitude of the ACF. In FCS, this can be avoided by selecting low-expressing cells or controlling the level of fluorescence by employing a photoactivatable GFP (paGFP), where a subset of tagged proteins can be activated prior to FCS measurements. Photoactivatable proteins yield robust FCS results ${ }^{94}$ but are not a strict requirement. It is important to note that they cannot be used for eN\&B because the dark species would artificially reduce the brightness $B$ value. The diffusion measurements and eN\&B should thus be carried out using the same standard (monomeric) fluorescent protein.

Most confocal microscopes have the capability of performing both FCS and N\&B measurements. A number of commercial platforms now provide FCS modules, including Zeiss and Olympus, which will automatically compute the ACF curve and provide mobility coefficients. Alternatively, raw data can be analyzed through a number of ImageJ plugins (https://imagej.nih.gov/ij/download.html) ${ }^{95}$ or SimFCS (Gratton Lab, University of California Irvine: https://www.Ifd.uci.edu/globals). Using a paGFP-tagged EphB2 we previously used established protocols ${ }^{28,94}$ to determine that the most appropriate camera exposure time is 500 ms when using the Zen FCS module of a Zeiss 780 platform (see Box 2 for a detailed procedure).

Once the protein mobility coefficient $\delta$ and the focal volume waist $\left(\omega_{0}\right)$ are known for the protein of interest (Box2), these together with the following guidelines can help the reader to choose the optimal acquisition parameters. The average time a protein remains in a focal volume (pixel), also known as residence time, can be computed as $\omega_{0}{ }^{2} / 4 \delta^{40}$. For camera-based microscopes, the time to take a single whole frame, $t_{\text {frame, }}$ depends mainly on the camera technical specifications, such as readout rate, number of pixels per frame and exposure (or dwell) time, $t_{d w e l l}$, needed to collect the protein fluorescence signal. When analyzing proteins with small mobility coefficients $\left(0.03-0.04 \mu \mathrm{m}^{2} / \mathrm{s}\right)$ with a fast camera (10 MHz, 512x512 pixels) readout time is approximately $26 \mathrm{~ms}$, $\mathrm{t}_{\mathrm{dwell}}(500 \mathrm{~ms})$ is almost equivalent to $\mathrm{t}_{\text {frame }}(526 \mathrm{~ms})$. Therefore, in these systems, to capture fluctuations (particles moving in and out of the focal volume) the exposure time is selected in such a way that $t_{\text {frame }}>\omega_{0}{ }^{2} / 4 \delta$ to allow the proteins to scatter through several pixels. If the sample is bright enough, the $t_{\text {frame }}$ can be increased simply by pausing between every acquisition ${ }^{1}$. This avoids averaging out fluctuations and may increase the statistical significance of the fluctuations. For laser-scanning microscopes, the dwell time 
$t_{d w e l l}$ is the time to collect the fluorescence signal at a single pixel and $t_{\text {frame }}$ depends then on the number of pixels $p$ as $t_{\text {frame }} \geq p t_{d w e l l}$. In these microscopes, $t_{d w e l l}$ should be shorter than $\omega_{0}{ }^{2 / 4} 4 \delta$ to avoid averaging out the fluctuations, i.e. to reduce the probability of a particle entering or exiting the focal volume; and $t_{\text {frame }}$ should be long enough to observe particle fluctuations ( $t_{\text {frame }}$ $\left.>\omega_{0}{ }^{2 / 4} \delta>p t_{d w e l l}\right)$. Therefore, $t_{d w e l l}$ and $t_{\text {frame }}$ can readily be selected for a particular microscope configuration if $\delta$ is approximately known.

Acquisition framework. Once the cells are ready to be imaged, the acquisition starts by capturing 200 sequential frames with an exposure time per frame that is proportional to the diffusion rate of the protein, as determined by FCS, Raster image correlation spectroscopy $\left(\right.$ RICS) ${ }^{96}$ or equivalent (Steps 13-18). The camera exposure time will determine the interval between time-points: since the 200 frames are treated as a single time-point, the longer the exposure time, the longer will take to capture the 200 frames. For short exposure times, many positions can be recorded at approximately the same time, for longer exposure times, the time to return to the same position may make the intervals between time-points too large in order to properly resolve the dynamics of the desired protein. Switching between positions needs to be done manually unless a custom macro is set up for every specific microscope system. If automatization is not possible, time annotation must be done manually at the beginning of every time point acquisition. This requires the presence of the researcher for the entire duration of the acquisition. For continuous imaging we advise dividing the acquisition into contiguous badges of 200 frames and treat them as individual time points.

eN\&B Analysis. We developed a user-friendly software package to perform eN\&B which makes use of an intuitive interface (Steps 19-27). The software and an example of the dataset analyzed during this study are available at http://bioimaging.usc.edu (see also Supplementary Video 1). Our software can be used to extract brightness values from fluorescence fluctuations for time-lapse image sequences. The code currently requires data to be organised as multiple multilayer stacks of images acquired at different time points, where a single file contains a sequence of images (see Experimental Design "Acquisition Framework" and Figure 5a). The software can perform two types of analysis, (i) full statistical resampling, which performs windowed-frame analysis on each of the time-point image sequences, providing a distribution of oligomerization states for each pixel, or (ii) a single-value analysis where only the mean value of oligomerization is reported. The full statistical resampling (i) performs eN\&B analysis enhancing the statistical resolution of the method at the expense of a longer computational time. The single-value analysis (ii) can be used to perform a rapid overview analysis of the experiment.

The software uses LOCI Bio-Formats ${ }^{97}$ to load microscopy data (Nikon proprietary file format in our case). In an effort to simplify adoption of the technique, we have created a Tiff file importer. The user can convert proprietary file formats (e.g. Olympus, Zeiss or Leica) to Tiff sequences prior to performing analysis. If Tiff file sequences are used, the number of frames per time point and the total number of time-points need to be specified at the software interface. 
An image of the time-series is then prompted to the user with the purpose of selecting an ROI in the field of view (Figure $5 b$ ). This allows for selective analysis of specific cells and including part of the background for reference during analysis. In the resulting scatter plot, each pixel of the image is represented in terms of intensity and Brightness (Figure $5 c, d$ ). The portion in the ROI related to background will generally provide a cluster at lower intensity values, while the sample will be shifted toward higher intensities. Manually selecting the boundary between these clusters is necessary for ensuring correct calculation of oligomerization levels.

Output data. The eN\&B software produces a series of images, plots and datasheets containing the measurements from the brightness analysis (Figure 6):

Materials

\section{BIOLOGICAL MATERIALS}

- Raw 16-bit TIFF grayscale images of the selected cell for every time point after photobleaching detrending. Only the first of the 200 frame series is shown (Figure 6a).

- The oligomerization maps show color-coded images of the cells with every pixel color coded (jet) on a scale according to the average oligomer size present in each pixel. Different oligomer binning options are presented to enhance oligomer populations contained in a narrow range of sizes (Figure 6b). Each binning option corresponds to differently equalized colormaps focusing on smaller, medium-sized or larger oligomers, or just evenly representing them and saved as 16-bit Tiff and png.

- $\quad \mathrm{i}$-mer plots display the time evolution of up to 40-mer oligomers (Figure 6c). These values are provided for multiple tolerances (sigma) around the value of the monomer.

- The abundance distribution of oligomers accumulated for all pixels in the image per every time point for the single-value analysis (Figure 6d) or the statistically-enhanced (Figure 6e). Of note, these distributions are not normalized by the total amount of pixels, therefore the integral of the distribution grows with the cell size. Raw data is also provided to handle data independently.

- Excel files containing image-histogram sum and percentage data from eN\&B analysis are provided. The values include the total number of pixels inside the selected ROI that are at a specific oligomerization level per time-point. These values are provided for multiple tolerances (sigma) around the value of monomer. Different files are provided for the quantification of the monomer to 40-mer range or the monomer to 100 -mer range.

- The full eN\&B file can be saved as a Matlab (.mat) file and includes the oligomerization distribution of every pixel for every time point.

- Cells expressing fluorescent proteins: in the example described in this protocol, we used the HEK293T:EphB2_mRuby cell line, which was generated by lentivirus transfection (ViraPower Lentiviral Packaging Mix, Thermo Fisher) of the plasmid pLenti.CMV:EphB2_mRuby. The plasmid pCDNA3_EphB2_mRuby was used as a source 
plasmid to excise the fusion construct. The cloning protocol is detailed in the original publication $^{28}$. The plamsids pLenti.CMV:EphB2 mRuby, and paGFP-EphB2are available upon request. Alternative generic genetic constructs for expression of fluorescent proteins, including mCherry or paGFP, can be obtained via Addgene.

- HEK293T cells were purchased directly to the distributor to avoid misidentification or cross-contamination (Sigma-Aldrich, cat. no. 85120602, ATCC $^{\circledR}$ CRL-3216 ${ }^{\mathrm{TM}}$ )

- Caution: regularly check your cells to avoid mycoplasma contamination and perform genetic tests and sequencing to ensure the cells are not crosscontaminated with different cell lines.

\section{REAGENTS}

- In-Fusion ${ }^{\circledR}$ HD Cloning Kit (Clontech, cat.no. 639606)

- ViraPower Lentiviral Packaging Mix (Thermo Fisher, cat.no. K497500)

- Caution: virus production must be carried out in a bio-safety level 2 laboratory or higher.

- DMEM (Sigma-Aldrich, cat. no. D6546-500ML)

- DMEM without phenol red (Thermo Fisher, cat. no. 21063-029)

- Fetal Bovine Serum (FBS - Hyclone, Cultek, S.L.U., cat. no. CH30160.03)

- Trypsin 0.05\% (wt/vol) (Thermo Fisher, cat. no. 25300-054)

- $\quad$ PBS, pH7.4 (Sigma-Aldrich, cat. no. P4417-100TAB )

- Lipofectamine 2000 (Thermo Fisher, cat. no. 11668-019)

- Opti Mem (Thermo Fisher, cat. no. 31985-047)

- Poly-L-lysine (Sigma-Aldrich, cat. no. P8920-100ML)

- Mili-Q water

- $\mathrm{NaOH}$ (sigma, cat. no. s0899)

- CAUTION: Causes severe skin burns and eye damage. Wear protective gloves and safety glasses.

- $\quad$ Fluorescent protein label, Atto 488 NHS ester (Atto-tec, product AD488-31)

\section{EQUIPMENT}

- 35 mm Glass bottom dishes (MatTek, cat. no.)

- $\quad$ LabTek glass bottom chamber slides, 1-well to 8-well (Lab-Tek ${ }^{\mathrm{TM}}$, Thermo Fisher)

- $\quad$ Engraved dishes (Zell-Kontakt).

- Acuderm Biopsy Punch 12 mm (Medex, cat. no. ACD-P1250)

- Vaccum Dessicator (Dynalon Labware, cat. no. 243025)

- Ultrasonic Bath (JP Selecta S.A., cat. no. 3000512)

- $\quad$ Shaker (Heidolph, cat. no. 543-42210-00)

- Table top centrifuge (Eppendorf, 5424R).

- Plate centrifuge (Eppendorf, 5810R)

- Rotator (Sturart, Rotator SB3) 
- Zeiss LSM 780 laser scanning confocal microscope equipped with Avalanche photodiodes of the Confocor 3 (Zeiss, Jena) equipped with a $405 \mathrm{~nm}$ laser line and a water $63 \mathrm{x} / 1.4$ NA objective (Zeiss, Jena)

- ZEN Software FCS and RICS modules (Zeiss, Jena)

- STORM microscope system (Nikon Instruments, NSTORM) equipped with an EMCCD camera (Andor iXon3 897), a 100x/1.4 NA objective and a 1.5x lens tube.

\section{Software}

- $\quad \operatorname{SimFCS~(www.Ifd.uci.edu);~}$

- FiJi (including Bio-Formats up-to-date plugin with ND2 files reader)https://fiji.sc/ ; https://loci.wisc.edu/software/bio-formats

- Matlab (The MathWorks, 2015 or newer)

(https://www.mathworks.com/products/matlab.html;

\section{Procedure}

\section{Setting up the microscope - TIMING $1 \mathrm{~h}$ to reach desired temperatures}

1. Warm up the microscope $1 \mathrm{~h}$ before starting the experiment to allow the temperature to stabilize, matching the sample optimal temperature (i.e. $37^{\circ} \mathrm{C}$ ). ?TROUBLESHOOTING

2. Turn on the $\mathrm{CO}_{2}$ and set up the controller at $5 \%$

\section{?TROUBLESHOOTING}

3. Turn on the camera $\sim 30 \mathrm{~min}$ before starting the experiment so that it reaches the optimal working temperature (i.e. $-70^{\circ} \mathrm{C}$ for an eMCCD ANDOR).

$\triangle$ CRITICAL STEP The camera read out is very sensitive to temperature oscillations.

4. Optional Step: Create a logbook text document on your PC. Clearly describe positions and time-points in the document (Table 1). This step is not necessary if your microscope allows automatic configuration of the imaging conditions.

5. Activate the relevant laser lines, allowing power sources to stabilize prior to image acquisition (in the example using our HEK293T:EphB2 mRuby cell line, we use the 561 $\mathrm{nm}$ laser). Set the laser power to a previously determined power density, which minimizes photobleaching (see Experimental Design "Instrument Calibration").

6. Ensure the light path is correctly specified:

- Dichroic mirrors and emission filters appropriate for the lasers and fluorophore being utilized, respectively.

- Additional magnification optics required to obtain the desired pixel size (106 nm here, see Experimental Design "Instrument Calibration"). 
7. Set the camera gain, readout to previously determined values (see Experimental Design "Gain" and "Camera readout mode").

8. Specify the number of $F$ frames per position and time point (i.e. $F=200$ )

9. Set the desired frame exposure time according to FCS measurements (see Experimental Design "Protein diffusion and camera exposure calibration").

10. Determine the optimal TIRF setting and proper illumination power. The objective here is to obtain high signal, low background images with low photobleaching rates. ?TROUBLESHOOTING

\section{Sample preparation - TIMING $30-45$ min}

11. Prepare the cells for imaging

i. Directly place the glass bottom MatTek or Labtek imaging chamber containing the cells of interest in the microscope.

ii. Choose a field of view containing 1 to 4 isolated cells. Record the position using the microscope software.

iii. Repeat Step ii until enough suitable positions have been recorded and select the best ones for imaging. ?TROUBLESHOOTING

iv. Gently pipette the ligand into the culture whilst avoiding moving the stage.

\section{Imaging $\odot$ TIMING $\mathbf{~ h ~}$}

12. Acquire a dark image sequence as follows: close the camera shutter and deactivate the lasers without turning them off. Acquire 200 frames with the shutter closed. Save this file and name it (i.e. dark_initial).

13. Focus the microscope on the cells in the first position, name the position file (i.e. Position1t1) (Figure 5a), start recording the 200 frames series and write down the starting time in the logbook.

?TROUBLESHOOTING

14. Immediately after finishing the first acquisition, move to the next position, refocus manually if necessary, name the file (i.e. Position2t1), start image acquisition and write down the starting time in the logbook.

15. Repeat Step 14 for as many positions as desired.

16. Switch back to the first position, name the file accordingly (i.e.Position1t2), start image acquisition and write down the starting time in the logbook. Repeat this step for all other positions.

17. Repeat Steps 14-16 for the duration of the entire imaging session. ?TROUBLESHOOTING

18. Close the camera shutter, record a dark frame series (see Step 12) and name it (i.e. dark_final).

Image analysis -TIMING 15 min per cell analyzed on N\&B mode, $\mathbf{2}$ h per cell on eN\&B mode

19. Run the eN\&B calibration, either as executable or as Matlab code (http://bioimaging.usc.edu): Load the sequence of single-tiff files containing the dark images. Record the parameters of the fit (Figure 3) for S factor, Sigma0, Offset and their precision 
20. Run the eN\&B GUI matlab code, either as executable or as Matlab code (http://bioimaging.usc.edu ) with the following parameters:

- Under Settings insert the numbers obtained from calibration from Step 19 and choose whether to show all figures or just to save them $\triangle$ CRITICAL STEP Image plotting is RAM memory expensive. If all images are shown upon calculation and less than 64GB RAM is available in the system, it is possible that the workstation runs out of memory before completion of the analysis.

- For fast N\&B analysis use the standard code

- For a statistically enhanced analysis use the eN\&B code.

21. Select the files from an entire time series from a single position ?TROUBLESHOOTING

22. Select a cell of interest by creating an ROI (Figure $5 b$ ). We suggest a physical size of the cell area to be analyzed larger than $64 \times 64$ pixels.

$\triangle$ CRITICAL STEP An optimal ROI should include a portion of the background outside the cell to provide a reference during analysis.

23. Double click on the cell ROI to complete the selection.

24. In the brightness scatter plot, establish the signal/noise threshold by marking the edge with the right end of the rectangle. If the selection of the ROI is performed correctly the plot should show easily distinguishable clusters (Figure $5 \mathrm{~d}$ ). The threshold should be placed at the lowest values of the right-most cluster, representing the cell. ?TROUBLESHOOTING

25. Double click on the corner of the ROI to trigger the analysis (Figure $5 \mathrm{~d}$ ). If all images are being shown in Step 20, the process can be observed until completion.

26. A file-save window will prompt requesting a root-name for saving the bulk files (images and excel files with raw data). The software saves by default the images in tiff and png formats. If further editing is required, save the individual images by clicking "saving as" on the relevant window (i.e .fig or .ems extensions).

27. Use the logbook data to specify the specific time-points values in the excel data (Table 1).

-TIMING

Steps 1-10, setting up the microscope: $1 \mathrm{~h}$ depending on the time needed by the camera and incubator to reach working temperature.

Step 11, sample preparation: 15 min to place the cells on the microscope and find regions of interest. Different samples may require different timing.

Steps $12-18$, imaging: $1 \mathrm{~h}$ or the specific time-course of the experiment.

Steps 19-27, image analysis: 15 min per cell on standard N\&B mode or additional 15 min if plot editing is required. $2 \mathrm{~h}$ per cell when running software on eN\&B mode.

\section{?TROUBLESHOOTING}




\section{$3 \quad$ Anticipated results}

4 The protocol detailed here can be used to image and quantify the oligomerization dynamics of

5 proteins. Fluorescently-tagged proteins are directly observed using a TIRF microscope during

6 serial image acquisitions. Imaging is carried out at the maximal resolution allowed by the

7 microscope set-up. The Number and Brightness (oligomerization) of the proteins in each pixel is

8 a function of the variance and intensity of the fluorescence fluctuations. The brightness values

9 for all pixels in a cell can be visualized as a color-coded oligomerization (brightness) value

10 overlaid with a cell image (Figure $6 b$ ). Since our eN\&B version includes algorithms minimizing

11 the impact of photobleaching, the method allows to resolve brightness maps during long time-

12 lapse imaging.

13 In addition to the oligomerization maps, the software can be used to retrieve more quantitative plots, which we termed i-mer plots (Figure $6 \mathrm{c}$ ). These plots display the evolution of the relative concentration of the different oligomers over time, displaying each oligomer species (up to 40-mer) as an independent curve. The value for each oligomer at a given time point results from the addition of the relative abundance of each oligomerization value (monomer, dimer etc.) from every pixel. In our experiments, the i-mer plots revealed a strikingly organized sequence of events, where progressively larger oligomers take over the smaller ones following a strict growth trajectory. Different ligand stimulations, such as soluble, surface immobilized or multivalent, substantially changed the trajectory of the different curves $^{28,62}$. For example, the slope of monomer depletion may reflect the speed of the oligomerization process. This plot is therefore the best tool to quantitative assess the clustering dynamics of a protein of interest.

The data contained in the i-mer plot can be also be presented from a population point of view. The relative abundance distribution of all oligomers at every time point is presented in plots like the one depicted in Figure $6 \mathrm{~d}$. This plot complements the i-mer plot, because the shape of every curve gives an overview of the diversity of the oligomer population present in the cell at every time point.

eN\&B uses a resampling method (what we call the enhancement) which procures per every time point an oligomerization distribution per every pixel, instead of a single value retrieved by the standard N\&B. This data obtained using eN\&B is too complicated to be represented in simple understandable plots without averaging the information. The plot in Figure 6e gives a rough idea of the amount of information generated by the method. The eN\&B data is better suited for additional mathematical or statistical analysis, rather than for graphic representation. The software generates matrixes containing all numerical values for that purpose. For every sample the relative abundance of every oligomer of every pixel in the image is included in an excel file or a Matlab matrix. This data is amenable for further mathematical analysis. The software allows to run the analysis on a standard N\&B mode, without performing the statistical enhancement. In this case the software will generate a single value per every pixel, corresponding with the modal value of the oligomerization distribution. Producing 
standard N\&B data is roughly 200 times faster than eN\&B data, and it is useful for exploratory analysis or qualitative observations.

We demonstrate our eN\&B using transgenic cells expressing a fluorescently-tagged EphB2 receptor. The cells were presented with ephrin ligands to induce receptor clustering during one hour time-course. The analysis was performed every 5 minutes to provide a detailed timecourse of Eph clustering. The oligomerization maps show Eph aggregation across the entire cell surface in a progressive manner (Figure $6 c, d$ ). Oligomerization runs uninterrupted during the entire time of observation. The i-mer plot shows a characteristic pattern which is repeated across many experiments. Monomers and low order oligomers can be seen to decay in the first 15 minutes after ligand addition. Thereafter, oligomers of progressively larger size increase their abundance in a strikingly coordinated pattern. An interesting feature of the EphB2 receptor is that clustering keeps running beyond the point of monomer depletion, which suggest that oligomers condense (coalesce) into larger ones. This type of behavior might not apply for other proteins and would need to be confirmed on a case by case basis. The shape of the oligomer distribution evolves over time as well (Figure $6 \mathrm{~d}$ ). At early time points narrow distributions center around small oligomer values. Over time, the center of the distribution shifts toward larger oligomers. The width of the distribution expands quickly over time, sometimes leading to long-tailed distributions, which reflects the growing diversity of the oligomer population also over time. The fast expansion of the distribution shape correlates with oligomer condensation. When using the enhanced version of the analysis it is advisable to analyze the same sample using the standard N\&B in parallel. In a qualitative way the results should be similar and the analysis runs faster. Plotted eN\&B data, such as in Figure $6 e$, can be challenging to read. For numerical analysis, the enhanced data contained in the Matlab matrixes provides a more complete and faithful description of the oligomer population.

\section{Acknowledgements}

S.O. research was supported by a Marie Curie International Outgoing Fellowship (276282) within the EU Seventh Framework Programme for Research and Technological Development (2007-2013), a postdoctoral fellowship from the Human Frontier Science Program Organization (LT000109/2011), and a postdoctoral fellowship (EX2009-1136) Ministerio de Educación from the Programa Nacional de Movilidad de Recursos Humanos del Plan Nacional de I-D+i 20082011. F.C. was supported by grants from the Moore Foundation and the NIH (R01 HD075605, R01 OD019037). J.J.O. acknowledges financial support from ICFONEST+, funded by the Marie Curie COFUND (FP7-PEOPLE-2010-COFUND) action of the European Commission and by the MINECO Severo Ochoa action at ICFO. Additional funding for this project came from Generalitat de Catalunya (2017-SGR-1079 and 2017-SGR-899); Spanish Ministry of Economy and Competitiveness (MINECO) (SAF2015-69706-R, MINAHE5, TEC2014-51940-C2-2-R, TEC2017-83716-C2-1-R; SEV-2015-0522); ISCIII/FEDER (RD16/0011/0024) EU (GLAM project, GA-634928; System's Microscopy Network of Excellence consortium,FP-7HEALTH.2010.2.1.2.2) and ERC (337191-MOTORS and 647863-COMIET), the Fundació Privada Cellex and CERCA Programme/Generalitat de Catalunya. The results presented here reflect only 
the views of the authors; the European Commission is not responsible for any use that may be made of the information it contains. The authors acknowledge the Nikon Center of

Excellence at ICFO.

\section{Author contributions}

S.O., J.J.O., A.S. and C.M. performed experiments. S.O., D.R., C.C. and F.C. analyzed the results and designed algorithms. V.H., E. L. and E.M. designed the micro-printing protocol. S.M. performed FCS analysis. M.L., E.M, A.R and S.E.F. contributed to the experimental design. S.O., F.C., J.J.O., C.C., D.R. and S.E.F. wrote the manuscript.

\section{Competing financial interests}

The authors declare no competing financial interests.

\section{Data/code availability statement}

The data collected for this study was done using our custom-made algorithms available at http://bioimaging.usc.edu. The data analysis for this study was done using our custom-made algorithms available at http://bioimaging.usc.edu.

\section{References}

1 Hartman, N. C. \& Groves, J. T. Signaling clusters in the cell membrane. Current opinion in cell biology 23, 370-376, doi:10.1016/j.ceb.2011.05.003 (2011).

2 Ali, M. H. \& Imperiali, B. Protein oligomerization: how and why. Bioorganic \& medicinal chemistry 13, 5013-5020, doi:10.1016/j.bmc.2005.05.037 (2005).

3 Marianayagam, N. J., Sunde, M. \& Matthews, J. M. The power of two: protein dimerization in biology. Trends in biochemical sciences 29, 618-625, doi:10.1016/j.tibs.2004.09.006 (2004).

4 Janes, P. W., Nievergall, E. \& Lackmann, M. Concepts and consequences of Eph receptor clustering. Seminars in cell \& developmental biology 23, 43-50, doi:10.1016/j.semcdb.2012.01.001 (2012).

5 Lemmon, M. A. \& Schlessinger, J. Cell signaling by receptor tyrosine kinases. Cell 141, 1117-1134, doi:10.1016/j.cell.2010.06.011 (2010).

6 Bray, D., Levin, M. D. \& Morton-Firth, C. J. Receptor clustering as a cellular mechanism to control sensitivity. Nature 393, 85-88, doi:10.1038/30018 (1998).

7 Nashmi, R. et al. Assembly of alpha4beta2 nicotinic acetylcholine receptors assessed with functional fluorescently labeled subunits: effects of localization, trafficking, and nicotine-induced upregulation in clonal mammalian cells and in cultured midbrain neurons. The Journal of neuroscience : the official journal of the Society for Neuroscience 23, 11554-11567 (2003).

8 Chiu, C. L. et al. Nanoimaging of focal adhesion dynamics in 3D. PloS one 9, e99896, doi:10.1371/journal.pone.0099896 (2014).

9 Digman, M. A., Wiseman, P. W., Choi, C., Horwitz, A. R. \& Gratton, E. Stoichiometry of molecular complexes at adhesions in living cells. Proceedings of the National Academy 
of Sciences of the United States of America 106, 2170-2175, doi:10.1073/pnas.0806036106 (2009).

10 Adu-Gyamfi, E. et al. A loop region in the N-terminal domain of Ebola virus VP40 is important in viral assembly, budding, and egress. Viruses 6, 3837-3854, doi:10.3390/v6103837 (2014).

11 Chiu, C. L., Digman, M. A. \& Gratton, E. Measuring actin flow in 3D cell protrusions. Biophysical journal 105, 1746-1755, doi:10.1016/j.bpj.2013.07.057 (2013).

12 Vishwasrao, H. D., Trifilieff, P. \& Kandel, E. R. In vivo imaging of the actin polymerization state with two-photon fluorescence anisotropy. Biophysical journal 102, 1204-1214, doi:10.1016/j.bpj.2012.01.031 (2012).

13 Lampe, M., Vassilopoulos, S. \& Merrifield, C. Clathrin coated pits, plaques and adhesion. Journal of structural biology 196, 48-56, doi:10.1016/j.jsb.2016.07.009 (2016).

14 Bhambhani, C., Chang, J. L., Akey, D. L. \& Cadigan, K. M. The oligomeric state of CtBP determines its role as a transcriptional co-activator and co-repressor of Wingless targets. The EMBO journal 30, 2031-2043, doi:10.1038/emboj.2011.100 (2011).

15 Khan, M. R. et al. Amyloidogenic Oligomerization Transforms Drosophila Orb2 from a Translation Repressor to an Activator. Cell 163, 1468-1483, doi:10.1016/j.cell.2015.11.020 (2015).

16 Marston, N. J., Jenkins, J. R. \& Vousden, K. H. Oligomerisation of full length p53 contributes to the interaction with mdm2 but not HPV E6. Oncogene 10, 1709-1715 (1995).

17 Hass, M. R. et al. SpDamID: Marking DNA Bound by Protein Complexes Identifies NotchDimer Responsive Enhancers. Molecular cell 64, 213, doi:10.1016/j.molcel.2016.09.035 (2016).

18 Schlierf, B., Ludwig, A., Klenovsek, K. \& Wegner, M. Cooperative binding of Sox10 to DNA: requirements and consequences. Nucleic acids research 30, 5509-5516 (2002).

19 Stein, E. et al. Eph receptors discriminate specific ligand oligomers to determine alternative signaling complexes, attachment, and assembly responses. Genes \& development 12, 667-678 (1998).

20 Hinde, E. et al. Quantifying the dynamics of the oligomeric transcription factor STAT3 by pair correlation of molecular brightness. Nature communications 7, 11047, doi:10.1038/ncomms11047 (2016).

21 Conway, A. et al. Multivalent ligands control stem cell behaviour in vitro and in vivo. Nature nanotechnology 8, 831-838, doi:10.1038/nnano.2013.205 (2013).

22 Salaita, K. et al. Restriction of receptor movement alters cellular response: physical force sensing by EphA2. Science 327, 1380-1385, doi:10.1126/science.1181729 (2010).

23 Dunsing, V., Mayer, M., Liebsch, F., Multhaup, G. \& Chiantia, S. Direct evidence of APLP1 trans interactions in cell-cell adhesion platforms investigated via fluorescence fluctuation spectroscopy. Molecular biology of the cell, doi:10.1091/mbc.E17-07-0459 (2017).

24 Plotegher, N., Gratton, E. \& Bubacco, L. Number and Brightness analysis of alphasynuclein oligomerization and the associated mitochondrial morphology alterations in live cells. Biochimica et biophysica acta 1840, 2014-2024, doi:10.1016/j.bbagen.2014.02.013 (2014).

25 Luna, E. \& Luk, K. C. Bent out of shape: alpha-Synuclein misfolding and the convergence of pathogenic pathways in Parkinson's disease. FEBS letters 589, 3749-3759, doi:10.1016/j.febslet.2015.10.023 (2015).

26 Cardenas-Aguayo Mdel, C., Gomez-Virgilio, L., DeRosa, S. \& Meraz-Rios, M. A. The role of tau oligomers in the onset of Alzheimer's disease neuropathology. ACS chemical neuroscience 5, 1178-1191, doi:10.1021/cn500148z (2014). 
27 Goedert, M. NEURODEGENERATION. Alzheimer's and Parkinson's diseases: The prion concept in relation to assembled Abeta, tau, and alpha-synuclein. Science 349, 1255555, doi:10.1126/science.1255555 (2015).

28 Ojosnegros, S. et al. Eph-ephrin signaling modulated by polymerization and condensation of receptors. Proceedings of the National Academy of Sciences of the United States of America 114, 13188-13193, doi:10.1073/pnas.1713564114 (2017).

29 Digman, M. A., Dalal, R., Horwitz, A. F. \& Gratton, E. Mapping the number of molecules and brightness in the laser scanning microscope. Biophysical journal 94, 2320-2332, doi:10.1529/biophysj.107.114645 (2008).

30 Digman, M. A., Dalal, R., Horwitz, A. F. \& Gratton, E. Mapping the number of molecules and brightness in the laser scanning microscope. Biophysical journal 94, 2320-2332, doi:10.1529/biophysj.107.114645 (2008).

31 Qian, H. \& Elson, E. L. On the analysis of high order moments of fluorescence fluctuations. Biophysical journal 57, 375-380, doi:10.1016/S0006-3495(90)82539-X (1990).

32 Qian, H. \& Elson, E. L. Distribution of molecular aggregation by analysis of fluctuation moments. Proceedings of the National Academy of Sciences of the United States of America 87, 5479-5483 (1990).

33 Moens, P. D., Gratton, E. \& Salvemini, I. L. Fluorescence correlation spectroscopy, raster image correlation spectroscopy, and number and brightness on a commercial confocal laser scanning microscope with analog detectors (Nikon C1). Microscopy research and technique 74, 377-388, doi:10.1002/jemt.20919 (2011).

34 Unruh, J. R. \& Gratton, E. Analysis of molecular concentration and brightness from fluorescence fluctuation data with an electron multiplied CCD camera. Biophysical journal 95, 5385-5398, doi:10.1529/biophysj.108.130310 (2008).

35 Hellriegel, C., Caiolfa, V. R., Corti, V., Sidenius, N. \& Zamai, M. Number and brightness image analysis reveals ATF-induced dimerization kinetics of UPAR in the cell membrane. FASEB journal : official publication of the Federation of American Societies for Experimental Biology 25, 2883-2897, doi:10.1096/fj.11-181537 (2011).

36 Hellriegel, C., Caiolfa, V. R., Corti, V., Sidenius, N. \& Zamai, M. Number and brightness image analysis reveals ATF-induced dimerization kinetics of UPAR in the cell membrane. FASEB journal : official publication of the Federation of American Societies for Experimental Biology 25, 2883-2897, doi:10.1096/fj.11-181537 (2011).

37 Trullo, A., Corti, V., Arza, E., Caiolfa, V. R. \& Zamai, M. Application limits and data correction in number of molecules and brightness analysis. Microscopy research and technique 76, 1135-1146, doi:10.1002/jemt.22277 (2013).

38 Ossato, G. et al. A two-step path to inclusion formation of huntingtin peptides revealed by number and brightness analysis. Biophysical journal 98, 3078-3085, doi:10.1016/j.bpj.2010.02.058 (2010).

39 Hur, K. H. et al. Quantitative measurement of brightness from living cells in the presence of photodepletion. PloS one 9, e97440, doi:10.1371/journal.pone.0097440 (2014).

40 Nolan, R. et al. Calibration-free In Vitro Quantification of Protein Homo-oligomerization Using Commercial Instrumentation and Free, Open Source Brightness Analysis Software. Journal of visualized experiments : JoVE, doi:10.3791/58157 (2018).

41 Nolan, R., Iliopoulou, M., Alvarez, L. \& Padilla-Parra, S. Detecting protein aggregation and interaction in live cells: A guide to number and brightness. Methods 140-141, 172177, doi:10.1016/j.ymeth.2017.12.001 (2018).

42 Adu-Gyamfi, E., Digman, M. A., Gratton, E. \& Stahelin, R. V. Investigation of Ebola VP40 assembly and oligomerization in live cells using number and brightness analysis. Biophysical journal 102, 2517-2525, doi:10.1016/j.bpj.2012.04.022 (2012). 
43 Hilsch, M. et al. Influenza A matrix protein M1 multimerizes upon binding to lipid

2

3

4

5

6

7

8

9

10

11

12

13

14

15

16

17

18

19

20

21

22 membranes. Biophysical journal 107, 912-923, doi:10.1016/j.bpj.2014.06.042 (2014).

44 Crosby, Kevin C. et al. Quantitative Analysis of Self-Association and Mobility of Annexin A4 at the Plasma Membrane. Biophysical journal 104, 1875-1885, doi:10.1016/j.bpj.2013.02.057 (2013).

45 James, Nicholas G. et al. in Biophysical journal Vol. 102 L41-L43 (2012).

46 Perumal, V., Krishnan, K., Gratton, E., Dharmarajan, A. M. \& Fox, S. A. in The International Journal of Biochemistry \& Cell Biology Vol. 64 91-96 (2015).

47 Youker, R. T. et al. Multiple motifs regulate apical sorting of p75 via a mechanism that involves dimerization and higher-order oligomerization. Molecular biology of the cell 24, 1996-2007, doi:10.1091/mbc.E13-02-0078 (2013).

48 Nagy, P., Claus, J., Jovin, T. M. \& Arndt-Jovin, D. J. Distribution of resting and ligandbound ErbB1 and ErbB2 receptor tyrosine kinases in living cells using number and brightness analysis. Proceedings of the National Academy of Sciences of the United States of America 107, 16524-16529, doi:10.1073/pnas.1002642107 (2010).

49 James, N. G. et al. A mutation associated with centronuclear myopathy enhances the size and stability of dynamin 2 complexes in cells. Biochimica et biophysica acta 1840, 315-321, doi:10.1016/j.bbagen.2013.09.001 (2014).

50 Labilloy, A. et al. Altered dynamics of a lipid raft associated protein in a kidney model of Fabry disease. Molecular Genetics and Metabolism 111, 184-192, doi:10.1016/j.ymgme.2013.10.010 (2014).

51 Olivera-Couto, A. et al. Eisosomes Are Dynamic Plasma Membrane Domains Showing Pil1-Lsp1 Heteroligomer Binding Equilibrium. Biophysical journal 108, 1633-1644, doi:10.1016/j.bpj.2015.02.011 (2015).

52 Ross, J. A. et al. in Biophysical journal Vol. 100 L15-L17 (2011).

53 Salvemini, I. L. et al. Low PIP2 molar fractions induce nanometer size clustering in giant unilamellar vesicles. Chemistry and Physics of Lipids 177, 51-63, doi:10.1016/j.chemphyslip.2013.11.003 (2014).

54 Presman, D. M. et al. DNA binding triggers tetramerization of the glucocorticoid receptor in live cells. Proceedings of the National Academy of Sciences of the United States of America 113, 8236-8241, doi:10.1073/pnas.1606774113 (2016).

55 Presman, D. M. et al. Live cell imaging unveils multiple domain requirements for in vivo dimerization of the glucocorticoid receptor. PLoS biology 12, e1001813, doi:10.1371/journal.pbio.1001813 (2014).

56 Abdisalaam, S., Davis, A. J., Chen, D. J. \& Alexandrakis, G. Scanning fluorescence correlation spectroscopy techniques to quantify the kinetics of DNA double strand break repair proteins after $\gamma$-irradiation and bleomycin treatment. Nucleic acids research 42, e5, doi:10.1093/nar/gkt908 (2014).

57 Vetri, V. et al. Fluctuation Methods To Study Protein Aggregation in Live Cells: Concanavalin A Oligomers Formation. Biophysical journal 100, 774-783, doi:10.1016/j.bpj.2010.11.089 (2011).

58 Mieruszynski, S., Briggs, C., Digman, M. A., Gratton, E. \& Jones, M. R. Live Cell Characterization of DNA Aggregation Delivered through Lipofection. Scientific reports $\mathbf{5}$, 10528, doi:10.1038/srep10528 (2015).

59 Kania, A. \& Klein, R. Mechanisms of ephrin-Eph signalling in development, physiology and disease. Nature reviews. Molecular cell biology 17, 240-256, doi:10.1038/nrm.2015.16 (2016).

60 Schaupp, A. et al. The composition of EphB2 clusters determines the strength in the cellular repulsion response. The Journal of cell biology 204, 409-422, doi:10.1083/jcb.201305037 (2014).

61 Klein, R. Eph/ephrin signalling during development. Development 139, 4105-4109, doi:10.1242/dev.074997 (2012). 
62 Hortiguela, V. et al. Nanopatterns of Surface-Bound EphrinB1 Produce Multivalent

2

3

4

5

6

7

8

9

10

11

12

13

14

15

16

17

18

19

20

21

22 Ligand-Receptor Interactions That Tune EphB2 Receptor Clustering. Nano letters 18, 629-637, doi:10.1021/acs.nanolett.7b04904 (2018).

63 Gambin, Y. et al. Confocal Spectroscopy to Study Dimerization, Oligomerization and Aggregation of Proteins: A Practical Guide. International journal of molecular sciences 17, doi:10.3390/ijms17050655 (2016).

64 Sahoo, B., Drombosky, K. W. \& Wetzel, R. Fluorescence Correlation Spectroscopy: A Tool to Study Protein Oligomerization and Aggregation In Vitro and In Vivo. Methods in molecular biology 1345, 67-87, doi:10.1007/978-1-4939-2978-8_5 (2016).

65 Herrick-Davis, K., Grinde, E., Lindsley, T., Cowan, A. \& Mazurkiewicz, J. E. Oligomer size of the serotonin 5-hydroxytryptamine $2 \mathrm{C}(5-\mathrm{HT} 2 \mathrm{C})$ receptor revealed by fluorescence correlation spectroscopy with photon counting histogram analysis: evidence for homodimers without monomers or tetramers. The Journal of biological chemistry 287, 23604-23614, doi:10.1074/jbc.M112.350249 (2012).

66 Krieger, J. W. et al. Imaging fluorescence (cross-) correlation spectroscopy in live cells and organisms. Nature protocols 10, 1948-1974, doi:10.1038/nprot.2015.100 (2015).

67 Chen, Y., Muller, J. D., So, P. T. \& Gratton, E. The photon counting histogram in fluorescence fluctuation spectroscopy. Biophysical journal 77, 553-567, doi:10.1016/S0006-3495(99)76912-2 (1999).

68 Muller, J. D., Chen, Y. \& Gratton, E. Resolving heterogeneity on the single molecular level with the photon-counting histogram. Biophysical journal 78, 474-486, doi:10.1016/S0006-3495(00)76610-0 (2000).

69 Caiolfa, V. R. et al. Monomer dimer dynamics and distribution of GPI-anchored uPAR are determined by cell surface protein assemblies. The Journal of cell biology 179, 1067-1082, doi:10.1083/jcb.200702151 (2007).

70 Srinivasan, R. et al. Forster resonance energy transfer (FRET) correlates of altered subunit stoichiometry in cys-loop receptors, exemplified by nicotinic alpha4beta2. International journal of molecular sciences 13, 10022-10040, doi:10.3390/ijms130810022 (2012).

71 Tosatto, L. et al. Single-molecule FRET studies on alpha-synuclein oligomerization of Parkinson's disease genetically related mutants. Scientific reports 5, 16696, doi:10.1038/srep16696 (2015).

72 Cremades, N. et al. Direct observation of the interconversion of normal and toxic forms of alpha-synuclein. Cell 149, 1048-1059, doi:10.1016/j.cell.2012.03.037 (2012).

73 Paredes, J. M. et al. Early amyloidogenic oligomerization studied through fluorescence lifetime correlation spectroscopy. International journal of molecular sciences 13, 94009418, doi:10.3390/ijms13089400 (2012).

74 Zanacchi, F. C. et al. A DNA origami platform for quantifying protein copy number in super-resolution. Nature methods 14, 789-792, doi:10.1038/nmeth.4342 (2017).

75 Hines, K. E. Inferring subunit stoichiometry from single molecule photobleaching. The Journal of general physiology 141, 737-746, doi:10.1085/jgp.201310988 (2013).

76 Youker, R. T. \& Teng, H. Measuring protein dynamics in live cells: protocols and practical considerations for fluorescence fluctuation microscopy. Journal of biomedical optics 19, 90801, doi:10.1117/1.JBO.19.9.090801 (2014).

77 Milo, R. \& Phillips, R. Cell biology by the numbers. (Garland Science, 2015).

78 Elowitz, M. B., Surette, M. G., Wolf, P. E., Stock, J. \& Leibler, S. Photoactivation turns green fluorescent protein red. Current biology : CB 7, 809-812 (1997).

79 Gambin, Y. et al. Lateral mobility of proteins in liquid membranes revisited. Proceedings of the National Academy of Sciences of the United States of America 103, 2098-2102, doi:10.1073/pnas.0511026103 (2006). 
2

3

4

5

6

7

8

9

10

11

12

13

14

15

16

17

18

19

20

21

22
80 Saffman, P. G. \& Delbruck, M. Brownian motion in biological membranes. Proceedings of the National Academy of Sciences of the United States of America 72, 3111-3113 (1975).

81 Rossing, T. Springer Handbook of Acoustics. (Springer New York, 2007).

82 Press, W. H., Teukolsky, S. A., Vetterling, W. T. \& Flannery, B. P. Numerical Recipes 3rd Edition: The Art of Scientific Computing. (Cambridge University Press, 2007).

83 Dalal, R. B., Digman, M. A., Horwitz, A. F., Vetri, V. \& Gratton, E. Determination of particle number and brightness using a laser scanning confocal microscope operating in the analog mode. Microscopy research and technique 71, 69-81, doi:10.1002/jemt.20526 (2008).

84 Wohland, T., Shi, X., Sankaran, J. \& Stelzer, E. H. Single plane illumination fluorescence correlation spectroscopy (SPIM-FCS) probes inhomogeneous three-dimensional environments. Optics express 18, 10627-10641, doi:10.1364/OE.18.010627 (2010).

85 Sisan, D. R., Arevalo, R., Graves, C., McAllister, R. \& Urbach, J. S. Spatially resolved fluorescence correlation spectroscopy using a spinning disk confocal microscope. Biophysical journal 91, 4241-4252, doi:10.1529/biophysj.106.084251 (2006).

86 Dempsey, G. T., Vaughan, J. C., Chen, K. H., Bates, M. \& Zhuang, X. Evaluation of fluorophores for optimal performance in localization-based super-resolution imaging. Nature methods 8, 1027-1036, doi:10.1038/nmeth.1768 (2011).

87 Ricci, M. A., Manzo, C., Garcia-Parajo, M. F., Lakadamyali, M. \& Cosma, M. P. Chromatin fibers are formed by heterogeneous groups of nucleosomes in vivo. Cell 160, 11451158, doi:10.1016/j.cell.2015.01.054 (2015).

88 Shaner, N. C., Steinbach, P. A. \& Tsien, R. Y. A guide to choosing fluorescent proteins. Nature methods 2, 905-909, doi:10.1038/nmeth819 (2005).

89 Kredel, S. et al. mRuby, a bright monomeric red fluorescent protein for labeling of subcellular structures. PloS one 4, e4391, doi:10.1371/journal.pone.0004391 (2009).

90 Shaner, N. C. et al. A bright monomeric green fluorescent protein derived from Branchiostoma lanceolatum. Nature methods 10, 407-409, doi:10.1038/nmeth.2413 (2013).

91 Elson, E. L. \& Magde, D. Fluorescence correlation spectroscopy. I. Conceptual basis and theory. Biopolymers 13, 1-27, doi:doi:10.1002/bip.1974.360130102 (1974).

92 Digman, M. A. \& Gratton, E. Lessons in fluctuation correlation spectroscopy. Annual review of physical chemistry 62, 645-668, doi:10.1146/annurev-physchem-032210103424 (2011).

93 Lakowicz, J. R. Principles of fluorescence spectroscopy. (2006).

94 Zhao, Z. W. et al. Quantifying transcription factor-DNA binding in single cells in vivo with photoactivatable fluorescence correlation spectroscopy. Nature protocols 12, 1458-1471, doi:10.1038/nprot.2017.051 (2017).

95 Schindelin, J. et al. Fiji: an open-source platform for biological-image analysis. Nature methods 9, 676-682, doi:10.1038/nmeth.2019 (2012).

96 Rossow, M. J., Sasaki, J. M., Digman, M. A. \& Gratton, E. Raster image correlation spectroscopy in live cells. Nature protocols 5, 1761-1774, doi:10.1038/nprot.2010.122 (2010).

97 Loci Bio-Formats, <https://loci.wisc.edu/software/bio-formats> (

98 Jaqaman, K. et al. Robust single-particle tracking in live-cell time-lapse sequences. Nature methods 5, 695-702, doi:10.1038/nmeth.1237 (2008).

99 Ovesny, M., Krizek, P., Borkovec, J., Svindrych, Z. \& Hagen, G. M. ThunderSTORM: a comprehensive ImageJ plug-in for PALM and STORM data analysis and super-resolution imaging. Bioinformatics 30, 2389-2390, doi:10.1093/bioinformatics/btu202 (2014).

100 Kaur, G. et al. Probing transcription factor diffusion dynamics in the living mammalian embryo with photoactivatable fluorescence correlation spectroscopy. Nature communications 4, 1637, doi:10.1038/ncomms2657 (2013). 


\section{FIGURE LEGENDS}

Figure 1. Enhanced Number and Brightness exploits fluorescence fluctuation analysis to extract the oligomerization state of proteins. a) During live-imaging, fluorescent molecules in different oligomerization states diffuse in and out of the focal volume. A sequence of $F$ images $(25 \leq \mathrm{F} \leq 200)$ can capture the fluorescence fluctuations derived from the movement of proteins into and out of the focal volume. As the size of the protein aggregate increases, the amplitude of the fluctuations increases over time while the period will decrease (see the red Eph receptor fluorescence fluctuation after ephrin stimulation and the control fluorescence from unstimulated cells). Kcps, kilo counts/s. b) For every time point ( $\mathrm{t} 1, \mathrm{t} 2, \mathrm{t} 3) \mathrm{N} \& \mathrm{~B}$ provides the mean oligomerization value for every pixel. eN\&B retrieves a distribution of species present in the same pixel, thus revealing a wider diversity of the oligomer population. c) Detrending algorithms correct the effect of photobleaching without affecting the fluorescence fluctuations. The image shows the acquisition of 100 frames from 3 different time points (initial, mid, final) from a total of $1 \mathrm{~h}$ imaging the EphB2 receptor. The average intensity (blue) decays exponentially, but the two representative pixels after correction (red and black) display intact fluctuations.

\section{Figure 2. Comparison of $\mathrm{EN \& B}$ and N\&B analysis of data from simulations of oligomers freely} diffusing in a liquid solution. The simulations were done with 1000 acquisition frames and an eN\&B window of 50 frames. In order to assess the power of analysis of eN\&B compared to standard N\&B, two opposed instances cover a scenario where a) monomers form oligomers over time and $\mathbf{b})$ multiple oligomeric states co-exist in parallel. The grey circles represent the size of the oligomer present at a given time. c-d) N\&B and eN\&B plots of the examples in a and b respectively. The single bar in the graph represents the mean oligomer value retrieved by standard N\&B. The solid line in the graph represents the oligomer size-distribution produced by eN\&B. mer, oligomer size. A.u., arbitrary units.

Figure 3. Analog Calibration for eN\&B analysis. a) Log scale Intensity distribution of a series of dark images, acquired in absence of sample, excitation and light, after allowing the instrument to temperature-stabilize (see section Experimental design "Analog Number and Brightness calibration"). b) The Gaussian component of the intensities histogram is used to obtain the offset (center) and readout noise (sigma). c) The exponential component slope fit provides the conversion factor $\mathrm{S}$ of intensity to photons. Fitting precision and parameters are reported next to each of the plots. d) Calibration is performed automatically by this tool, providing a final fit overview and the factor values needed for calibration of the system in the algorithm.

Figure 4. Typical ACF curve obtained from FCS analysis. The EphB2 receptor fused to a photoactivatable GFP (paGFP) was analyzed using FCS. The Normalized intensity correlation (G) shows a linear decay at a certain period range. The inverse of that period is the optimal camera exposure time.

Figure 5. eN\&B software interface and data loading. a) Files are selected from the folder and sorted in a time-sequential manner using the move up or move down buttons. Every file 


\section{Table 1. Example of a customized logbook for time-lapse registration}

plate 1, 0.2uM ephrinb1

position $1 \mathrm{t} 1=9.21$

position $2 \mathrm{t} 1=11.36$

position $1 \mathrm{t} 2=13.52$

position $2 \mathrm{t} 2=16.13$

(...)

position $1 \mathrm{t} 10=50.07$

position $2 \mathrm{t} 10=52.27$

Table 2. Troubleshooting Table

\begin{tabular}{|l|l|l|l|}
\hline Step & Problem & Possible reason & Solution \\
\hline 1 & $\begin{array}{l}\text { Cells show } \\
\text { unexpected behavior, } \\
\text { apoptosis, poor } \\
\text { attachment, etc. }\end{array}$ & $\begin{array}{l}\text { The temperature at the } \\
\text { sample illumination point } \\
\text { is not } 37 \stackrel{\circ}{ } \mathrm{C}\end{array}$ & $\begin{array}{l}\text { Most microscope incubators show very } \\
\text { heterogeneous temperature pattern across } \\
\text { the chamber, which may not match the } \\
\text { display settings in the temperature } \\
\text { controller. It is advisable to use a precise }\end{array}$ \\
\hline
\end{tabular}




\begin{tabular}{|c|c|c|c|}
\hline & & & $\begin{array}{l}\text { temperature probe placed in the sample } \\
\text { illumination point and tune the } \\
\text { temperature controller settings to reach } \\
\text { real } 37{ }^{\circ} \mathrm{C}\end{array}$ \\
\hline 2 & $\begin{array}{l}\text { My microscope does } \\
\text { not have } \mathrm{CO}_{2} \text { chamber } \\
\text { and the medium } \\
\text { acidifies quickly }\end{array}$ & $\begin{array}{l}\text { The } \mathrm{pH} \text { needs to be } \\
\text { buffered }\end{array}$ & $\begin{array}{l}\text { Switch to } \mathrm{L} 15 \text { media when a } \mathrm{CO}_{2} \text { chamber is } \\
\text { not present. It buffers in atmospheric } \\
\text { conditions unlike DMEM and other medias }\end{array}$ \\
\hline 10 & $\begin{array}{l}\text { The cells look more } \\
\text { blurry than usual, or } \\
\text { the signal is not as } \\
\text { sharp }\end{array}$ & $\begin{array}{l}\text { The TIRF angle needs to } \\
\text { be adjusted, as a result of } \\
\text { change of objective or } \\
\text { the use of a different } \\
\text { brand plate with different } \\
\text { thickness. }\end{array}$ & $\begin{array}{l}\text { For consistency it is preferred to use the } \\
\text { same plate type and brand as well as the } \\
\text { same objective }\end{array}$ \\
\hline 11 & $\begin{array}{l}\text { The cells look dim or } \\
\text { too bright (saturated } \\
\text { pixels) }\end{array}$ & $\begin{array}{l}\text { The microscope set up is } \\
\text { not optimal }\end{array}$ & $\begin{array}{l}\text { The parameters that allow you to obtain a } \\
\text { better signal are the illumination power, } \\
\text { camera gain, and exposure time. Increasing } \\
\text { the illumination power will result in higher } \\
\text { levels of photobleaching but will increase } \\
\text { the signal efficiently. Increasing the } \\
\text { exposure time will also result in higher } \\
\text { levels of photobleaching though the signal } \\
\text { increase will be smaller and will affect the } \\
\text { brightness analysis. Increasing the camera } \\
\text { gain will not affect photobleaching but it } \\
\text { will increase the noise. }\end{array}$ \\
\hline 11(iii) & $\begin{array}{l}\text { Finding suitable cells } \\
\text { takes too long }\end{array}$ & $\begin{array}{l}\text { Low cells were seeded } \\
\text { confluence } \\
\text { The cells change shape } \\
\text { and move }\end{array}$ & $\begin{array}{l}\text { Increasing the cell confluence will increase } \\
\text { the chance to find suitable cells for } \\
\text { imaging, and also the probability of having } \\
\text { more than one cell in the same field of } \\
\text { view. The software can analyze the cells } \\
\text { individually, so having more than one cell in } \\
\text { the same field of view will accelerate data } \\
\text { acquisition. } \\
\text { Try different coating (i.e. PLL, laminin, } \\
\text { gelatine) }\end{array}$ \\
\hline 13 & $\begin{array}{l}\text { The cell images are } \\
\text { dark }\end{array}$ & $\begin{array}{l}\text { The camera shutter is } \\
\text { closed }\end{array}$ & $\begin{array}{l}\text { Ensure to re-open the camera shutter after } \\
\text { acquiring the last dark frame }\end{array}$ \\
\hline 17 & $\begin{array}{l}\text { The cells move } \\
\text { The recorded } \\
\text { positions seem to } \\
\text { change between time- } \\
\text { points }\end{array}$ & $\begin{array}{l}\text { Cell motion (due to } \\
\text { migration, movements } \\
\text { from filopodia or similar } \\
\text { projections, cell } \\
\text { spreading etc.) may be } \\
\text { unavoidable and it } \\
\text { interferes with brightness } \\
\text { measurements. } \\
\text { The plate is drifting }\end{array}$ & $\begin{array}{l}\text { If the microscope allows recording several } \\
\text { positions simultaneously, we recommend } \\
\text { to capture as many cells as possible. Review } \\
\text { the movies at the end of the process to } \\
\text { discard the motile cells. The selection of a } \\
\text { proper adhesive coating will improve the } \\
\text { results. } \\
\text { Make sure you allow at least one hour for } \\
\text { the microscope to reach and stabilize at the } \\
\text { desired temperature. Excessive immersion } \\
\text { liquid will also increase the probability of }\end{array}$ \\
\hline
\end{tabular}




\begin{tabular}{|c|c|c|c|}
\hline & & & $\begin{array}{l}\text { drifting. Make sure the stage holds the } \\
\text { plate tightly. }\end{array}$ \\
\hline 25 & $\begin{array}{l}\text { The software crashes } \\
\text { before finishing the } \\
\text { analysis }\end{array}$ & $\begin{array}{l}\text { The computer is not } \\
\text { powerful enough for } \\
\text { eN\&B }\end{array}$ & $\begin{array}{l}\text { Find a computer with minimum 64GB RAM } \\
\text { memory available, enough hard drive space } \\
\text { available. }\end{array}$ \\
\hline 25 & $\begin{array}{l}\text { The software gives an } \\
\text { error message }\end{array}$ & $\begin{array}{l}\text { The error message } \\
\text { usually contains } \\
\text { information regarding the } \\
\text { problem. Possible } \\
\text { reasons include } \\
\text { insufficient memory for } \\
\text { visualizing or saving the } \\
\text { data, noise and } \\
\text { background signal have } \\
\text { not been separated } \\
\text { properly, file type being } \\
\text { loaded is incompatible }\end{array}$ & $\begin{array}{l}\text { Try unchecking the "Show all images" } \\
\text { checkbox in the eN\&B GUI Settings. Try } \\
\text { repeating the noise and background } \\
\text { selection with a higher threshold. Make } \\
\text { sure images are loaded either as Nikon .nd2 } \\
\text { files or as single TIFF files. }\end{array}$ \\
\hline 26 & $\begin{array}{l}\text { The oligomerization } \\
\text { maps are almost } \\
\text { empty }\end{array}$ & $\begin{array}{l}\text { Noise separation was too } \\
\text { stringent }\end{array}$ & $\begin{array}{l}\text { Repeat the analysis selecting a wider area } \\
\text { of signal pixels. }\end{array}$ \\
\hline
\end{tabular}

1

2

3

4

\section{5}

6

7

8

9

1. Treat a LabTek chambered glass slide with $1 \mathrm{M} \mathrm{NaOH}$ for 10-15 minutes and let it air dry.

2. Dilute a fluorescently labelled protein/antibody in PBS to approximately 0.1-1 $\mathrm{ng} / \mathrm{ml}$.

CRITICAL STEP Prepare a sample of fluorescently labelled proteins/antibodies using a fluorophore in the same spectral range as will be utilized in the experimental project (i.e. ATTO 488 for GFP).

3. Incubate the diluted solution onto the glass surface for 2-5 minutes.

4. Wash thoroughly with PBS.

5. Image the prepared sample to ensure that individual fluorescent proteins/antibodies can be visualized as isolated bright spots on a dark background. 
CRITICAL STEP If too many/too few individual spots are visualized, repeat sample preparation and adjust the protein/antibody dilution or the incubation time.

6. Record several hundred frames, imaging the single molecules over a wide range of EM gain settings to image the single molecules as they gradually photobleach.

CRITICAL STEP We suggest as starting range: $10-1000$ gain in log-scale intervals (i.e. 10, 30, 100, 300, 1000). Recommended exposure time: 30-50 ms.

7. Perform single-particle tracking of the spots by fitting each of them with a $2 \mathrm{D}$ Gaussian function with constant offset.

CRITICAL STEP Several open source softwares are available to do this, such as u-track ${ }^{98}$ or ThunderSTORM ${ }^{99}$. Note the latter will require that localizations in each frame be linked together to obtain a fluorescence trajectory for each single protein/antibody imaged.

8. Remove the contribution from background fluorescence prior to fitting. CRITICAL STEP If the software used does not provide this quantity (background count) as an output, obtain it by subtracting the number of background photons/counts from the integrated number of photons/counts.

9. For each EM gain setting, first calculate the mean and standard deviation of the background subtracted photon/count values for each single-molecule fluorescence trajectory. Second, calculate the ratio of mean to standard deviation for the fluorescence trajectory. The maximal value of this ratio is the optimized EM gain setting.

10. If camera settings allow different pixel transfer rates, repeat steps 6-9 at different readout speeds (i.e. $1 \mathrm{MHz}, 5 \mathrm{MHz}, 10 \mathrm{MHz}$ ).

\section{- END OF BOX 1 -}

\section{Box 2. Applying FCS to quantify protein mobility coefficient and define camera exposure settings}

This Box describes a protocol to carry out FCS using photoactivatable proteins. This approach allows to extract protein diffusion coefficients $(\delta)$ efficiently. $\delta$ values are required to calibrate the camera exposure time during eN\&B imaging. The detailed original protocol can be found in Ref. ${ }^{94}$.

\section{Procedure:}

CRITICAL: The focal volume waist $\left(\omega_{0}\right)$ and structural parameter $(S)$ must first be calibrated using a fluorophore with a known diffusion coefficient such as eGFP, FITC or certain Alexa/ATTO probes of the relevant emission channel (Steps 1-3). 
1. Place a drop of $\sim 1 \mathrm{nM}$ solution of Atto488 in water onto a glass coverslip directly over the water $\times 63 / 1.4$ NA objective of a Zeiss 780 (Zeiss, Jena). At $25^{\circ} \mathrm{C}$ this small molecule has a known diffusion coefficient of $400 \mu \mathrm{m}^{2} \mathrm{~s}^{-1}$.

2. Within the Zen FCS module (Zeiss, Jena), acquire data fluorescence counts of the Atto 488 standard at a single point on the $488 \mathrm{~nm}$ laser line for $25 \mathrm{~s}$ with 4 repeats. The physical conditions (temperature, molarity, buffers) must be consistent to the conditions for the known diffusion coefficient of the fluorophore in use.

3. The Atto488 should contain a single species freely difusing. Within the "Fit" tab, fit the acquired data to the single free diffusion component model provided within the FCS module with a fixed diffusion coefficient of $400 \mu \mathrm{m}^{2} \mathrm{~s}^{-1}$, and variable volume $\omega_{0}$ and $\mathrm{S}$. The software will compute these two parameters based on the Atto 488 standard, which are essential when determining and unknown diffusion coefficient. Record the calculated volume $\omega_{0}$ and $S$ parameters. A detailed step-by-step protocol provided with the Zeiss FCS module and is a freely available online resource from Zeiss (https://www.zeiss.com/content/dam/Microscopy/Downloads/Pdf/FAQs/zen2010Ism780_basic_fcs_experiments.pdf).

4. Seed cells onto glass bottom chamber slides and transiently transfect them with a paGFP-tagged EphB2 and membrane-mCherry vector (Addgene:53750) using Lipofectamine 2000 according to the manufacturer's guidelines. The amount of DNA transfected and the DNA:lipid ratio should be optimised based on the cell line used. CRITICAL STEP When using a paGFP fused protein it is recommended to co-transfect with a constitutively fluorescent marker to identify points of interest in the cell.

4. Mount the cells on the microscope and identify transfected cells based on the expression of membrane-mCherry.

5. To activate the paGFP, draw a region-of-interest along the membrane using the membrane-mCherry as a guide. Activate the paGFP using the $405 \mathrm{~nm}$ laser line, with the laser power and dwell line optimized for each cell and level of transfection:. The GFP signal should be visible, but the fluorescence still sparse.

CRITICAL STEP: Activating a small defined region will reduce phototoxicity and experimental time.

6. Within the Zen FCS module, select the point of acquisition with the crosshairs options using the mCherry reference to identify the membrane. In our setup, the FCS measurement of EphB2-paGFP was carried out with the $488 \mathrm{~nm}$ laser line by acquiring $4 \times 25 \mathrm{~s}$ cycles of data collection. Multiple cycles were collected as the membrane may ruffle in and out of focus slightly, so the best of the four cycles was analyzed.

CRITICAL STEP: Measuring small structures such as membranes can be difficult, therefore the application of scanning-FCS may be desirable.

7. Fit the FCS data to a previously published 2 -species model ${ }^{100}$. This provides the ACF and in our case resulted in the identification of two rates of motion at 0.03 and $0.04 \mu^{2} \mathrm{~s}^{-1}$ at regions with and without cell-to-cell contact, respectively.

\section{- END OF BOX 2 -}

\section{SUPPLEMENTARY INFORMATION}


1 - Supplementary Video: Screen recording of the analysis of a single cell using eN\&B software

2 - Supplementary Figure 1: Example of photobleaching detrending on cells that were not

3 stimulated with the ligand shows consistent brightness correction 